\title{
Direct imaging of small scatterers using reduced time dependent data
}

\author{
Fioralba Cakoni*and Jacob D. Rezac ${ }^{\dagger}$
}

\begin{abstract}
We introduce qualitative methods for locating small objects using time dependent acoustic near field waves. These methods have reduced data collection requirements compared to typical qualitative imaging techniques. In particular, we only collect scattered field data in a small region surrounding the location from which an incident field was transmitted. The new methods are partially theoretically justified and numerical simulations demonstrate their efficacy. We show that these reduced data techniques give comparable results to methods which require full multistatic data and that these time dependent methods require less scattered field data than their time harmonic analogs.
\end{abstract}

\section{Introduction}

A primary goal of inverse scattering theory is the reconstruction of information about unknown objects based on how acoustic or electromagnetic waves scatter off of them. These problems have applications in nondestructive testing and identifying hidden objects (e.g., underground material resources or defects in structures). A popular class of methods for solving these problems, called qualitative methods, are able to quickly and accurately determine the shape and location of hidden objects, and require little a priori information about the objects. They are non-iterative in nature and do not require large scale wave simulations. We refer the reader to [12] for a comprehensive account of these methods for inhomogeneous media.

Though effective, qualitative methods typically require a large amount of scattered field data to be collected: the typical multistatic set-up is for scattered field data to be collected at all points on a ball surrounding the unknown object, corresponding to incident field data emitted from all points on the same ball. In this paper, we propose two schemes which significantly reduce the amount of data required for accurate reconstructions. In both schemes, we use a small array of transmitters and receivers constructed so that data is collected only in a small region. Incident waves are emitted from the transmitters, collected by the

\footnotetext{
*Department of Mathematics, Rutgers University, Piscataway, NJ 08854-8019, USA (fc292@math.rutgers.edu)

$\dagger$ Department of Mathematical Sciences, University of Delaware, Newark, DE 19716, USA (jrezac@udel.edu)
} 
nearby receivers, and the entire device is moved to a new location where the experiment is repeated. In one scheme, we allow the device to contain many transmitters and receivers, collecting multistatic data only in patches with a small aperture. In the other scheme, a quasi-backscattering set-up, the array contains one transmitter and a small number of receivers in a small neighborhood of the transmitter. We must increase the amount of a priori information we assume about the object in order to justify these methods theoretically. Particularly, we will assume objects are small and weakly scattering.

The quasi-backscattering data collection scheme proposed here is somewhat similar to the time harmonic study initiated in [26] and studied further in [24], though the reconstruction method and applicability of the method here differs. Of particular importance here is that the algorithms described below directly use causal time dependent near field data and require no Fourier or Laplace transformation into frequency domain data. In many applications, ranging from medical imaging to non-destructive testing, time dependent data is readily obtained. Moreover, as our numerical examples will demonstrate, using time dependent data allows us to use significantly fewer transmitters and receivers than time harmonic data. Most previous studies of similar problems make use of time harmonic far field data with one or multiple frequencies. In some applications, far field data is required due to physical constraints on how near to an object sensors can be placed. Nonetheless, near field data is sometimes easier to obtain in practice, and typically results in higher resolution reconstructions. Furthermore, the type of data collection scheme suggested here is readily implementable in practice. For example, a device with transmitters and receivers concentrated in a small region was built in [17] to collect scattered field data for potential industrial applications.

To make the above comments precise, assume scattering is caused by time dependent acoustic waves propagating through a medium with a variable speed of sound, $c \in L^{\infty}\left(\mathbb{R}^{d}\right)$ $(d=2$ or 3$)$ so that $c(x) \geq \gamma>0$ for some $\gamma \in \mathbb{R}$. We assume a constant background speed of sound, $c_{0}=1$. Let $u^{i}(x, t ; y)$ indicate the incident field emitted from a point $y \in \mathbb{R}^{d}$ evaluated at a point $x \in \mathbb{R}^{d} \backslash\{y\}$ and time $t \in \mathbb{R}^{+}$. Such an incident field satisfies the free space acoustic wave equation,

$$
u_{t t}^{i}-\Delta_{x} u^{i}=0 \quad \text { for } x \in \mathbb{R}^{d} \backslash\{y\}, \quad t \in \mathbb{R}^{+} .
$$

The resulting scattered field, $u^{s}(x, t ; y)$, satisfies

$$
\begin{array}{ll}
c^{-2}(x) u_{t t}^{s}-\Delta u^{s}=-\left(c^{-2}(x)-1\right) u_{t t}^{i} & (x, t) \in \mathbb{R}^{d} \times \mathbb{R}^{+} \\
u^{s}(x, 0)=u_{t}^{s}(x, 0)=0 \quad x \in \mathbb{R}^{d} . &
\end{array}
$$

Define $n(x)=c^{-2}(x)$ to be the index of refraction and $D=\operatorname{supp}(1-n(x))$ to be the location of the unknown scatterer. We will be more precise about $n$ and $D$ below. Let $\chi \in \mathcal{C}^{2}(D)$ be a causal temporal pulse function (that is, $\chi$ and its derivatives vanish for $t<0$ ). We define the incident field originating at a point $y \in \mathbb{R}^{d}$ as the time convolution of $\chi$ with the fundamental solution $\Phi$ to the wave equation,

$$
\Phi(x, t)= \begin{cases}\frac{H(t-|x|)}{2 \pi \sqrt{t^{2}-|x|^{2}},} & d=2, \\ \frac{\delta(t-|x|)}{4 \pi|x|}, & d=3,\end{cases}
$$


where $H$ is the Heaviside function. For example, in $\mathbb{R}^{3}$

$$
u^{i}(x, t ; y)=\frac{\chi(t-|x-y|)}{4 \pi|x-y|}, \quad(x, t) \in\left(\mathbb{R}^{3} \backslash y\right) \times \mathbb{R}^{+} .
$$

The inverse problem is to find $D$ from $u^{s}(x, t ; y)$ for $x \in \Gamma_{m}, y \in \Gamma_{i}, t \in \mathbb{R}^{+}$where the measurement and incident locations, $\Gamma_{m}$ and $\Gamma_{i}$ respectively, are sets in $\mathbb{R}^{d-1}$ which do not intersect with $D$. For example, the typical full aperture multistatic set-up is $\Gamma_{m}=\Gamma_{i}=$ $\partial B_{R}(0)$, where $\partial B_{R}(0)$ is the boundary of a ball of radius $R>0$ centered at the origin where $R$ is large enough that $D \subset B_{R}(0)$. In the limited aperture case $\Gamma_{m}, \Gamma_{i} \subset \partial B_{R}(0)$ (possibly $\Gamma_{m}=\Gamma_{i}$ ). See Figure 1 (left) for a sample of a limited aperture multistatic geometry.

In this paper, we will primarily use reduced data. First, we use a series of limited aperture multistatic arrays which are moved around the obstacles. For example, let $\Gamma_{i}=\Gamma_{m}$ be patches with a small area in $\mathbb{R}^{3}$. We collect multistatic data with this patch and then move the entire array to a new location and collect data again. The second type of reduced data is a quasi-backscattering set-up. To describe this data set-up, let $\Gamma_{i} \subset \mathbb{R}^{d-1}$ be the curve on which we will place transmitting devices. We again assume we can collect data only with a small device which moves around $\Gamma_{i}$. Denote by $\delta>0$ a small constant. For each fixed $y \in \Gamma_{i}$, data is collected on $\Gamma_{m}^{(y)}:=\Gamma_{i} \cap B_{\delta}(y)$, where $B_{R}(x)$ is the ball of radius $R>0$ centered at $x \in \mathbb{R}^{d}$. See Figure 1 (right) for a sample set-up geometry in $\mathbb{R}^{2}$. Note that this set-up requires more data than the related backscattering data, in which each transmitter has just one associated receiver, and both are located at the same point.
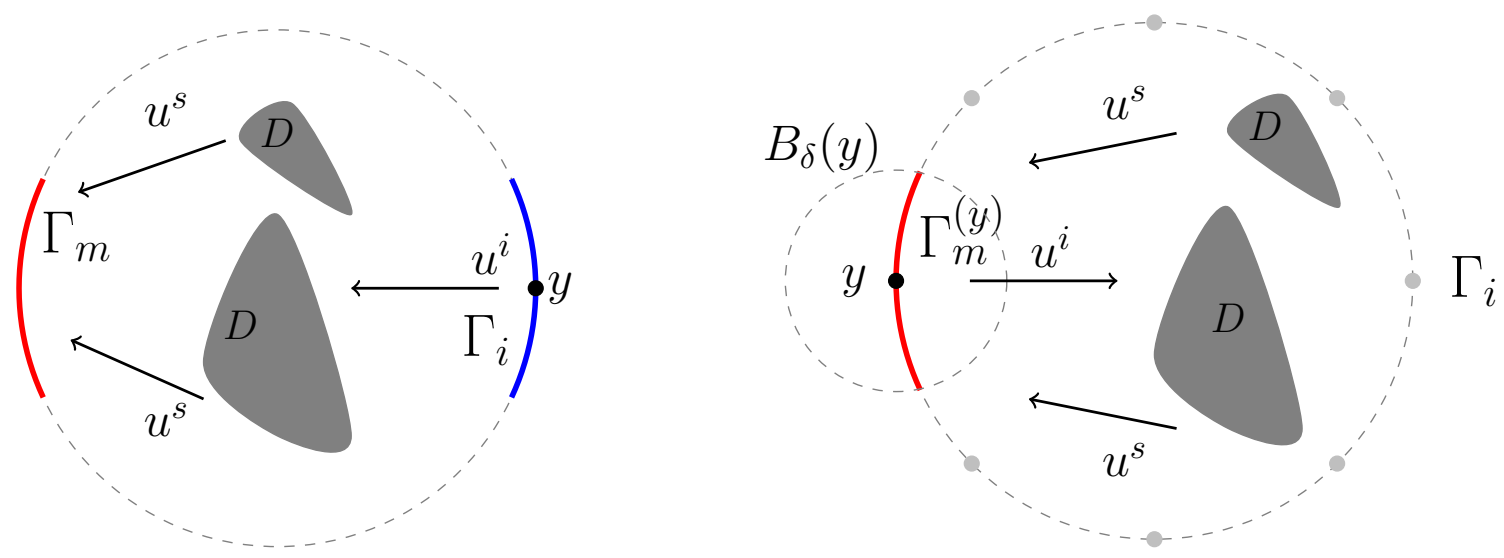

Figure 1: Examples of limited aperture multistatic (left) and quasi-backscattering (right) measurements. In the limited aperture multistatic figure, the blue line represents the location of transmitters and the red line the location of receivers. In the quasi-backscattering set-up, $\Gamma_{i}$ is the large dashed circle, the thick solid line is $\Gamma_{m}^{(y)}$ for a fixed $y \in \Gamma_{i}$, and the circles on $\Gamma_{i}$ not located at $y$ represent locations to which $\Gamma_{m}^{(y)}$ will be moved.

There exist many qualitative methods for solving inverse scattering problems with multistatic data. In [20, 21], a qualitative method known as the linear sampling method is used to approximate the shape of $D$ using causal multistatic time-domain scattering data. In these papers, the theoretical justification of the method remains incomplete due to technical problems involving an associated problem called the time domain interior transmission 
problem. This will be discussed in more detail in Section 3.4. This is in contrast to the time domain linear sampling method for scattering from bounded objects with Dirichlet, Neumann, or Robin boundary conditions whose theory is fully described in $[14,25]$. The multifrequency linear sampling method, which can be seen as time dependent technique with non-causal waves, is studied in [22]. In [32,35], it was shown that, under certain conditions, a potential function related to speed of sound can be calculated based on backscattered time domain data collected in the far field. While these require less data than we do, they solve a slightly different problem than we do here and do not provide a method for constructing the potential. Time reversal methods, described for example in the review article [16], are also popular for solving inverse scattering problems with time dependent data.

The time harmonic backscattering problem for small and weak scatterers was studied in [18] using multiple frequencies. The reduction of data collection requirements in the time harmonic case is studied from a different perspective in [2]. The study of time harmonic scattering from small and weak scatterers is extensive. One popular class of techniques (see, e.g., $[4,6,8,9,15,28])$ are called MUltiple SIgnal Classification (MUSIC) techniques. Such imaging methods typically require multistatic data and operate by testing if a particular indicator function related to a point in space is in the range of an operator related to the collected data. In this article, we also introduce a MUSIC-type algorithm which directly makes use of time dependent near field data, which has some similarities to the time domain MUSIC algorithm for small bounded objects introduced in [3]. For an introduction to multistatic time harmonic approaches to qualitative methods in inverse scattering problems, see the monographs $[5,12,28]$.

A number of recent reconstruction algorithms have been proposed, e.g. [1, 19, 31], which reduce data requirements by using only one incident source and scattered field data with receivers surrounding the objects. Such approaches result in fast data collection, since there is only one experiment required, but require that the objects can be simultaneously surrounded by receivers. We take a different approach to data reduction here, assuming there is enough time to perform many experiments, but that the objects cannot be completely surrounded by receivers at the same time. This approach is useful in the case of imaging large regions or in cases where it is costly to place many receivers at once.

\section{Forward Model and the Born Approximation}

We begin by discussing the well-posedness of (1). This is well known, and to discuss it precisely we follow $[11,20,30]$, introducing some space-time Sobolev spaces described through the Fourier-Laplace transform. This will allow us to introduce and state the well-posedness of a time domain weak scattering approximation and its frequency domain counterpart. This approximation is related to the well-known Born approximation and so we will refer to it as such. These will be used in Section 3.1 to validate a multistatic MUSIC-type algorithm in the time domain.

As discussed in the introduction, we are able to reduce the amount of data required for reconstructions by making a priori assumptions on the contrast $n$ and the scatterer $D$. In particular, we will make a weak scattering Born approximation in which multiple scattering effects are minor and can be ignored. Assume that $n(x)=1+\epsilon m(x)$ for $\epsilon \ll 1, m=O(1)$, and 
that solutions to (1) take the form $u^{s}(x, t)=u_{0}^{s}(x, t)+\epsilon u_{B}^{s}(x, t)$. The function $u_{B}^{s}=\left.\frac{\partial u^{s}}{\partial \epsilon}\right|_{\epsilon=0}$ is the well-known Born approximation. Indeed, if $u^{i}(x, t)$ is of the same order as $u_{0}^{s}(x, t)$ for $x \in D$, then separating into powers of $\epsilon$ yields $u_{0}^{s} \equiv 0$ and $u_{B}^{s}$ satisfies

$$
\begin{array}{ll}
\frac{\partial^{2} u_{B}^{s}}{\partial t^{2}}-\Delta u_{B}^{s}=-m \frac{\partial^{2} u^{i}}{\partial t^{2}} & \text { for }(x, t) \in \mathbb{R}^{d} \times \mathbb{R}^{+} \\
u_{B}^{s}(x, 0)=\frac{\partial u_{B}^{s}}{\partial t}(x, 0)=0 & \text { for } x \in \mathbb{R}^{d} .
\end{array}
$$

This time domain Born approximation should be considered as a linearization of the scattered field with respect to the strength of scatterers, rather than as the first term of a series solution to (1) in the way that the time harmonic Born approximation sometimes is; as is discussed in Remark 4.5 of [29], terms associated with higher order terms in $\epsilon$ are not necessarily well-defined in any reasonable spaces.

To solve (3), we take a Green's function approach and take a space-time convolution of $m(x) u_{t t}^{i}$ with $\Phi(x, t)$. This results in a time domain Lippmann-Schwinger equation. For $f \in \mathcal{C}_{0}^{\infty}(D \times \mathbb{R})$, define the retarded volume potential operator $V$ by

$$
(V f)(x, t):=\int_{\mathbb{R}} \int_{D} \Phi(x-z, t-\tau) f(\tau, z) \mathrm{d} V(z) \mathrm{d} \tau, \quad(x, t) \in \mathbb{R}^{d} \times \mathbb{R}
$$

where $\Phi(x, t)$ is given by $(2)$. Then, $v(x, t)=(V f)(x, t)$ satisfies

$$
v_{t t}-\Delta v=f \quad \text { in } \mathbb{R}^{d} \times \mathbb{R} .
$$

Later we will also use the related single layer potential, $S_{\Gamma}$, defined by

$$
\left(S_{\Gamma} f\right)(x, t):=\int_{\mathbb{R}} \int_{\Gamma} \Phi(x-y, t-\tau) f(\tau, y) \mathrm{d} s(y) \mathrm{d} \tau, \quad(x, t) \in\left(\mathbb{R}^{d} \backslash \Gamma\right) \times \mathbb{R}
$$

where $\Gamma$ is some closed surface.

In order to make these equations precise, we recall the appropriate space-time Sobolev spaces, following $[20,30]$. To this end, we first introduce the Fourier-Laplace transform. Let $\omega=\eta+i \sigma$ for $\eta, \sigma \in \mathbb{R}$ with $\sigma>\sigma_{0}>0$ for some $\sigma_{0} \in \mathbb{R}$. We use the notation $\mathbb{C}_{\sigma_{0}}=\left\{\omega \in \mathbb{C}: \operatorname{Im}(\omega) \geq \sigma_{0}>0\right\}$ to define this half-plane. Let $X$ be a Hilbert space. The set of temporal, smooth, and compactly supported in $[0, \infty) X$-valued functions is denoted by $\mathcal{D}\left(\mathbb{R}^{+} ; X\right)=C_{0}^{\infty}(\mathbb{R} ; X)$. The associated $X$-valued distributions on the real line which vanish for time $t<0$ are denoted by $\mathcal{D}^{\prime}\left(\mathbb{R}^{+} ; X\right)$ and the corresponding tempered distributions by $\mathcal{S}^{\prime}\left(\mathbb{R}^{+} ; X\right)$. Define

$$
\mathcal{L}_{\sigma}^{\prime}\left(\mathbb{R}^{+}, X\right):=\left\{f \in \mathcal{D}^{\prime}\left(\mathbb{R}^{+}, X\right): e^{-\sigma t} f \in \mathcal{S}^{\prime}\left(\mathbb{R}^{+}, X\right), \text { for some } \sigma(f)<\infty\right\}
$$

to be the space of functions with well-defined Fourier-Laplace transforms. Indeed, the Fourier-Laplace transform of $f=f(x, t) \in \mathcal{L}_{\sigma}^{\prime}\left(\mathbb{R}^{+}, X\right)$, denoted by $\hat{f}(x, \omega)$ is given by

$$
\hat{f}(x, \omega)=\int_{0}^{\infty} f(x, t) \exp (i \omega t) \mathrm{d} t, \quad \omega \in \mathbb{C}_{\sigma_{0}}
$$

for $\sigma_{0}=\sigma_{0}(f)$ and $x \in \mathbb{R}^{d}, t \in \mathbb{R}$. 
Note that $\hat{f}(x, \omega)=\mathcal{F}\left(e^{-\sigma t} f\right)(\eta)$ where $\mathcal{F}$ represents the typical Fourier transform on causal functions, so many properties of the Fourier transform will transfer to the FourierLaplace transform with little change. We can now define the Hilbert space for $p \in \mathbb{N}_{0}$, $\sigma \in \mathbb{R}$,

$$
H_{\sigma}^{p}\left(\mathbb{R}^{+}, X\right):=\left\{f \in \mathcal{L}_{\sigma}^{\prime}\left(\mathbb{R}^{+} ; X\right): \int_{\mathbb{R}+i \sigma}|\omega|^{2 p}\|\hat{f}(\cdot, \omega)\|_{X}^{2} d s<\infty\right\} .
$$

By Parseval's theorem, the norm of this space is equivalent to

$$
\|f\|_{H_{\sigma}^{p}\left(\mathbb{R}^{+} ; X\right)}^{2}=\int_{0}^{\infty} e^{-2 \sigma t}\left\|\frac{\partial^{p} f(\cdot, t)}{\partial t^{p}}\right\|_{X}^{2} \mathrm{~d} t
$$

where we have used the fact that $f$ and its derivatives vanishes for $t<0$. For more details see e.g. [23]. With this notation in hand, we have the following result about the solvability of (3), where $\sigma>\sigma_{0}>0$ for a $\sigma_{0}$ depending on the specifics of the problem.

Theorem 1 ([30], Theorem 3.2). For $r=0,1,2$ and $p \in \mathbb{R}, V: H_{\sigma}^{p}\left(\mathbb{R}^{+}, L^{2}(D)\right) \rightarrow$ $H_{\sigma}^{p+1-r}\left(\mathbb{R}^{+}, H^{r}\left(\mathbb{R}^{d}\right)\right)$ is a bounded linear operator. Moreover, if $v=V(f)$ for some $f \in$ $H_{\sigma}^{p}\left(\mathbb{R}^{+}, L^{2}(D)\right)$ then $v(t)=0$ for $t<0$ and $v \in H_{\sigma}^{p}\left(\mathbb{R}^{+}, H^{1}\left(\mathbb{R}^{d}\right)\right)$ satisfies

$$
v_{t t}-\Delta v=f \quad \text { in } H_{\sigma}^{p-1}\left(\mathbb{R}^{+}, L^{2}\left(\mathbb{R}^{d}\right)\right) .
$$

Theorem 1 allows us to write

$$
\begin{aligned}
u_{B}^{s}(x, t ; y) & =-\left(V m u_{t t}^{i}\right)(x, t) \\
& =-\int_{\mathbb{R}} \int_{D} m(z) \Phi(x-z, t-\tau) u_{t t}^{i}(z, \tau ; y) \mathrm{d} V(z) \mathrm{d} \tau, \quad(x, t) \in \mathbb{R}^{d} \times \mathbb{R}^{+} .
\end{aligned}
$$

For later, we introduce the bounded linear solution operator for (3),

$$
G: H_{\sigma}^{p}\left(\mathbb{R}^{+}, L^{2}(D)\right) \rightarrow H_{\sigma}^{p+1-r}\left(\mathbb{R}^{+}, H^{r}\left(\mathbb{R}^{d}\right)\right) .
$$

which takes $u^{i}$ to $u_{B}^{s}$ with (4). Here $\sigma, p$, and $r$ are as in Theorem 1. From the above, the solution of the Born wave equation satisfies

$$
\left\|u_{B}^{s}\right\|_{H_{\sigma}^{p}\left(\mathbb{R}^{+}, H^{1}\left(\mathbb{R}^{d}\right)\right)} \leq C\left\|u^{i}\right\|_{H_{\sigma}^{p}\left(\mathbb{R}^{+}, L^{2}(D)\right)} .
$$

See also [34] for a discussion of these properties in both $\mathbb{R}^{2}$ and $\mathbb{R}^{3}$.

Taking the Fourier-Laplace transform of $V$ gives an equivalent formulation in the frequencydomain. In particular, for $f \in \mathcal{C}_{0}^{\infty}(D)$ define the operator $\hat{V}$ by

$$
(\hat{V} f)(x ; \omega)=\int_{D} \hat{\Phi}_{\omega}(x, z) f(z) \mathrm{d} V(z), \quad x \in \mathbb{R}^{d}
$$

where $\hat{\Phi}_{\omega}(\cdot, \cdot)$ is the fundamental solution of the Helmholtz equation with wavenumber $\omega \in$ $\mathbb{C}_{\sigma_{0}}$,

$$
\hat{\Phi}_{\omega}(x, z):=\left\{\begin{array}{lll}
\frac{i}{4} H_{0}^{(1)}(\omega|x-z|) & d=2, & \\
\frac{e^{i \omega|x-z|}}{4 \pi|x-z|} & d=3 & x \neq z
\end{array}\right.
$$


for some $\sigma_{0}>0$. Here $H_{0}^{(1)}$ is the Hankel function of order zero of the first kind. It can be shown [30] that $\hat{V}: L^{2}(D) \rightarrow H^{2}\left(\mathbb{R}^{d}\right)$ and that if $\hat{v}=\hat{V} f$ then $\hat{v}$ satisfies

$$
\Delta \hat{v}+\omega^{2} \hat{v}=f \quad \text { in } \mathbb{R}^{d} .
$$

Hence,

$$
\Delta \hat{u}_{B}^{s}+\omega^{2} \hat{u}_{B}^{s}=-\omega^{2} m \hat{u}^{i}
$$

has the solution

$$
\hat{u}_{B}^{s}(x, \omega ; y)=-\omega^{2} \int_{D} m(z) \hat{\Phi}_{\omega}(x, z) \hat{u}^{i}(z ; y) \mathrm{d} V(z) .
$$

If $\operatorname{Im}(\omega)=0$ then the Fourier-Laplace transform becomes the standard Fourier transform, (8) becomes the usual equation for a Born approximation to the time harmonic scattered field with wavenumber $\operatorname{Re}(\omega)$, and (9) is the first term of the Born series.

\section{Inverse problem for the time domain Born approxi- mation}

We now discuss time dependent imaging algorithms, two for multistatic data and one for quasi-backscattering data. We first introduce a MUSIC-type method for imaging weak and small scatterers which is fully justified theoretically. As a specific case of this method, we describe a reconstruction algorithm using multistatic patch data. Next, we introduce a MUSIC-type method for quasi-backscattering data. As the numerical results in Section 4 suggest, both techniques can be used to find obstacles from time domain data. Finally, we discuss the linear sampling method for extended weak scatterers with multistatic data and why it lacks full justification.

Each algorithm we develop below takes a similar form, as indicated by the general algorithm below. In particular, we define an indicator function, depending on $z \in \mathbb{R}^{d}$ (and possibly on time $\tau \in[0, T]$ for some $T>0$ ), so that the function is large when $z \in D$ and small otherwise. As such, the bulk of this section is dedicated to deriving indicator functions and demonstrating that they are large when $z \in D$.

\begin{tabular}{ll}
\hline & Algorithm Frequency (time) domain sampling methods for reconstruction of obstacles \\
\hline Step 1 & Collect scattered field data at $x \in \Gamma_{m}, y \in \Gamma_{i}($ and $t \in[0, T])$. \\
Step 2 & Select sampling grid points $\mathcal{Z}$ (and $\tau \in[0, T])$. \\
Step 3 & Plot the indicator function for each $z \in \mathcal{Z}($ and $\tau \in[0, T])$. \\
Step 4 & Post-process or regularize the indicator function to assert the collection of $z \in D$. \\
\hline
\end{tabular}

The primary idea behind each of these indicator functions is that a specified function, which depends on $z$, is in the range of an operator depending on $u^{s}$ if and only if $z \in D$. For multistatic data, we are interested in the near field equation

$$
\left(N_{\text {multi }} g_{z, \tau}\right)(y, t)=\ell_{z, \tau}^{\xi}(y, t), \quad(y, t) \in \Gamma_{i} \times \mathbb{R}^{+}
$$


for each $z \in \mathcal{Z}$, where the near field operator $N_{\text {multi }}: L_{\sigma}^{2}\left(\mathbb{R}^{+}, L^{2}\left(\Gamma_{m}\right)\right) \rightarrow L_{\sigma}^{2}\left(\mathbb{R}^{+}, L^{2}\left(\Gamma_{i}\right)\right)$, $\sigma>0$ is defined by

$$
\left(N_{\text {multi }} g\right)(y, t)=\int_{\mathbb{R}} \int_{\Gamma_{m}} u_{B}^{s}(x, t-\tau ; y) g(x, \tau) \mathrm{d} s(x) \mathrm{d} \tau, \quad(y, t) \in \Gamma_{i} \times \mathbb{R}^{+} .
$$

In Section 3.4 below we will give more details about the mapping properties of $N_{\text {multi }}$.

Furthermore,

$$
\ell_{z, \tau}^{\xi}(y, t):=\int_{\mathbb{R}} \Phi\left(y-z, t-\tau-t_{0}\right) \xi\left(t_{0}\right) \mathrm{d} t_{0}
$$

is the convolution of a smooth compactly supported $\xi \in C_{c}^{\infty}(\mathbb{R})$ with the fundamental solution of the wave equation given by (2). For example, in $\mathbb{R}^{3}, \ell_{z, \tau}^{\xi}(y, t)=\frac{\xi(t-\tau-|y-z|)}{4 \pi|y-z|}$. The idea for quasi-backscattering data is similar.

\subsection{Reconstruction of point scatterers from time domain multi- static data}

Assume now that $D$ is composed of $M$ weak point scatterers located at the points $z_{j} \in \mathbb{R}^{d}$, $j=1, \ldots, M$. Let the contrast $m$ be of the form $m(x)=\sum_{j=1}^{M} m_{j} 1_{D_{j}}(x)$ where $m_{j}$ are constant. In this section we collect multistatic data and introduce a MUSIC-type algorithm for locating small objects based on near field time domain data. Hence, let $\Gamma_{i} \subset \mathbb{R}^{d-1}$ be the curve from which incident fields are transmitted and let $\Gamma_{m} \subset \mathbb{R}^{d-1}$ be the curve on which the resulting scattered field is measured. We assume the curves do not intersect $D$ and that they are either closed curves or open subsets of analytic curves. Below we will take $\Gamma_{i}=\Gamma_{m}$.

In the above configuration, the near field operator (11) takes the form

$$
\left.\left(N_{\text {multi }} g\right)(y, t)=-\sum_{j=1}^{M} m_{j}\left(\Phi\left(z_{j}-y, \cdot\right) *\left(\mathcal{S}_{\Gamma_{m}}^{\ddot{\chi}} g\right)\right)\left(z_{j}, \cdot\right)\right)(t), \quad(y, t) \in \Gamma_{i} \times \mathbb{R}^{+},
$$

where

$$
\left(S_{\Gamma_{m}}^{\ddot{\chi}} g\right)(x, t)=\left(\ddot{\chi}(\cdot) *\left(S_{\Gamma_{m}} g\right)(x, \cdot)\right)(t)
$$

and $*$ indicates a time convolution. Hence, the point scattering near field equation (10) becomes

$$
\left.-\sum_{j=1}^{M} m_{j}\left(\Phi\left(z_{j}-y, \cdot\right) *\left(\mathcal{S}_{\Gamma_{m}}^{\ddot{\chi}} g\right)\right)\left(z_{j}, \cdot\right)\right)(t)=\ell_{y, \tau}^{\xi}(y, t), \quad(y, t) \in \Gamma_{i} \times \mathbb{R}^{+} .
$$

The Fourier-Laplace transform $N_{\text {multi }}$ in this point scattering context yields the frequency domain weakly scattering near field operator, $\widehat{N}_{\text {multi }}: L^{2}\left(\Gamma_{m}\right) \rightarrow L^{2}\left(\Gamma_{i}\right)$ defined as

$$
\left(\widehat{N}_{\text {multig }}\right)(y, \omega)=-\sum_{j=1}^{M} m_{j} \widehat{\ddot{\chi}}(\omega) \hat{\Phi}_{\omega}\left(z_{j}, y\right) \int_{\Gamma_{m}} \hat{\Phi}_{\omega}\left(x, z_{j}\right) \hat{g}(x, \omega) \mathrm{d} s(x), \quad \omega \in \mathbb{C}_{\sigma_{0}}
$$

for some $\sigma_{0}>0$. 
Similarly, the Fourier-Laplace transform of $\ell_{z, \tau}^{\xi}$ is

$$
\widehat{\ell_{z, \tau}^{\xi}(y, t)}(y, \omega)=\hat{\xi}(\omega) e^{i \omega \tau} \hat{\Phi}_{\omega}(y, z),
$$

where $\hat{\Phi}_{\omega}$ is given by (7). Thus the transformed point scattering near field equation reads

$$
\sum_{j=1}^{M} \alpha_{j} \hat{\Phi}_{\omega}\left(y, z_{j}\right)=\beta \hat{\Phi}_{\omega}(y, z), \quad y \in \Gamma_{i}, z \in \mathbb{R}^{d}
$$

where $\alpha_{j}=-m_{j} \widehat{\ddot{\chi}}(\omega) \int_{\Gamma_{m}} \hat{\Phi}_{\omega}\left(x, z_{j}\right) \hat{g}(x, \omega) \mathrm{d} s(x), \beta=\hat{\xi}(\omega) e^{i \omega \tau}$ are constants depending on $\hat{g}$, $z_{j}, \tau, m_{j}, \Gamma_{m}$, and $\omega$. Point scattering approximations of the type derived here can also be derived through high frequency truncation of asymptotic expansions, as explained in [7].

For fixed $\omega, \tau=0$, and $\hat{\xi}(\omega) \equiv 1$, (16) leads the MUSIC algorithm in the frequency domain; the following lemma allows us to characterize the range of $\hat{N}_{\text {multi }}$.

Lemma 1. Let $\Gamma_{i}$ be a closed curve or an open subset of an analytic curve and let $z_{j} \in$ $\mathbb{R}^{d}, j=1, \ldots, M$ be distinct points which do not lie on $\Gamma_{i}$. Then the sets of functions $\left\{y \mapsto \hat{\Phi}_{\omega}\left(y, z_{j}\right): j=1, \ldots, M, y \in \Gamma_{i}\right\}$ are linearly independent for any $\omega \in \mathbb{C}_{\sigma_{0}}$ with $\sigma_{0}=\sigma_{0}(\Phi)>0$.

Proof. Let $a_{j} \in \mathbb{C}$ be constants so that

$$
\sum_{j=1}^{M} a_{j} \hat{\Phi}_{\omega}\left(y, z_{j}\right)=0 \quad y \in \Gamma_{i} .
$$

Since $\hat{\Phi}_{\omega}(y, z)$ are solutions to the Helmholtz equation, they are real analytic on $y$ away from $y=z$. Without loss of generality, assume $\Gamma_{i}$ is a closed curve. Otherwise, we can analytically continue (17) to the analytic curve of which $\Gamma_{i}$ is a subset.

Note that the left hand side of (17) is a radiating solution to the Helmholtz equation outside of $\Gamma_{i}$. Hence, by unique continuation and uniqueness of the exterior Dirichlet problem with boundary $\Gamma_{i}$, (17) is in fact true for all $y \in \mathbb{R}^{d} \backslash\left\{z_{1}, z_{2}, \ldots, z_{M}\right\}$. Due to the singularity of $\hat{\Phi}_{\omega}(y, z)$ at $y=z$, letting $y \rightarrow z_{j}$, we see that each $a_{j}$ must vanish identically.

The above lemma enables us to characterize the range of the finite rank operator $\hat{N}_{\text {multi }}$ which in turn leads to a test to locate the point scatterers. Note that the proof of this theorem is very similar to the proof of the equivalent theorem for far field data given in [28]. We include this proof for completeness.

Theorem 2. Assume $\omega \in \mathbb{C}_{\sigma_{0}}$ for some $\sigma_{0}>0, D=\left\{z_{1}, z_{2}, \cdots z_{M}\right\}$ and that $\Gamma_{i}$ and $\Gamma_{m}$ (not necessarily the same) are closed curves or open subsets of analytic curves which do not intersect $D$. Then $\hat{\Phi}_{\omega}(\cdot, z) \in \operatorname{Range}\left(\hat{N}_{\text {multi }}\right)$ if and only if $z=z_{j}$ some $j=1, \ldots, M$.

Proof. Assume by contradiction that $z=z_{0} \notin\left\{z_{1}, \ldots, z_{M}\right\}$, and that there is some $g \in$ $L^{2}\left(\Gamma_{m}\right)$ so that $\left(\hat{N}_{\text {multi }} g\right)(y)=\hat{\Phi}_{\omega}\left(y, z_{0}\right)$ for each $y \in \Gamma_{i}$. By definition of $\hat{N}_{\text {multi }}$,

$$
\hat{\Phi}_{\omega}\left(y, z_{0}\right)=\sum_{j=1}^{M} \alpha_{j} \hat{\Phi}_{\omega}\left(y, z_{j}\right), \quad y \in \Gamma_{i} .
$$


This is a contradiction with the linear independence shown in Lemma 1. Hence, if $\hat{\Phi}_{\omega}(\cdot, z) \in$ Range $\left(\hat{N}_{\text {multi }}\right)$ then $z \in\left\{z_{1}, z_{2} \ldots, z_{M}\right\}$.

Now assume $z \in\left\{z_{1}, z_{2}, \ldots, z_{M}\right\}$. We will show that $\hat{\Phi}_{\omega}(\cdot, z) \in \operatorname{Kern}\left(\hat{N}_{\text {multi }}^{*}\right)^{\perp}=$ $\operatorname{Range}\left(\hat{N}_{\text {multi }}\right)$ (notice that $\hat{N}_{\text {multi }}$ is finite rank so $\overline{\operatorname{Range}\left(\hat{N}_{\text {multi }}\right)}=\operatorname{Range}\left(\hat{N}_{\text {multi }}\right)$ ). We explicitly calculate the adjoint of $\hat{N}_{\text {multi }}^{*}: L^{2}\left(\Gamma_{i}\right) \rightarrow L^{2}\left(\Gamma_{m}\right)$ as

$$
\left(\hat{N}_{\text {multi }}^{*} h\right)(x)=-\sum_{j=1}^{M} m_{j} \overline{\hat{\ddot{\chi}}(\omega) \hat{\Phi}_{\omega}\left(x, z_{j}\right)} \int_{\Gamma_{i}} \overline{\hat{\Phi}_{\omega}\left(y, z_{j}\right)} h(y) \mathrm{d} s(y) .
$$

As such, if $h \in \operatorname{Kern}\left(\hat{N}_{\text {multi }}^{*}\right)$ then

$$
\sum_{j=1}^{M} m_{j} \overline{\hat{\ddot{\chi}}(\omega) \hat{\Phi}_{\omega}\left(x, z_{j}\right)} \int_{\Gamma_{i}} \overline{\hat{\Phi}_{\omega}\left(y, z_{j}\right)} h(y) \mathrm{d} s(y)=0, \quad \text { for } x \in \Gamma_{m}
$$

and by the assumption on $\Gamma_{m}$, the linear independence shown in Lemma 1 gives

$$
0=\int_{\Gamma_{i}} \hat{\Phi}_{\omega}\left(y, z_{j}\right) \overline{h(y)} \mathrm{d} s(y) .
$$

Hence each $\hat{\Phi}_{\omega}\left(\cdot, z_{j}\right) \in \operatorname{Kern}\left(\hat{N}_{\text {multi }}^{*}\right)^{\perp}=\operatorname{Range}\left(\hat{N}_{\text {multi }}\right)$.

While we have primarily proven Theorem 2 in order to prove a similar result for the time dependent case, it also gives a MUSIC-type inversion scheme for multistatic weakly scattering near field time harmonic data. In particular, both Lemma 1 and Theorem 2 follow in an identical way for real positive values of $\omega \in \mathbb{R}, \omega>0$. Then $u^{s}$ is time harmonic acoustic scattering data from a point source incident field. Let $P_{\hat{N}_{\text {multi }}^{\perp}}: L^{2}\left(\Gamma_{i}\right) \rightarrow \mathcal{R}\left(\hat{N}_{\text {multi }}\right)^{\perp}$ be the orthogonal projection onto the orthogonal complement of the range of $\hat{N}_{\text {muti }}$. Theorem 2 gives that $P_{\hat{N}_{\text {multi }}^{\perp}} \hat{\Phi}_{\omega}(\cdot, z)=0$ if and only if $z=z_{j}$.

In a typical MUSIC application, the function $I(z)=\left\|P_{\hat{N}_{\text {multi }}^{\perp}} \hat{\Phi}_{s}(y, z)\right\|_{L^{2}\left(\Gamma_{i}\right)}$ serves as an indicator function to locate $D$ : if $D \subset \mathcal{Z}$ for some set of sampling points $\mathcal{Z} \subset \mathbb{R}^{d}$, then $(I(z))^{-1}$ is large for each $z \in D$ and small otherwise. We change this slightly here and test the angle between Range $\left(\hat{N}_{\text {multi }}\right)$ and $\hat{\Phi}_{\omega}(y, z)$ for each $z \in \mathcal{Z}$. When the angle between these is very small, we assert that $\hat{\Phi}_{\omega}(y, z) \in \operatorname{Range}\left(\hat{N}_{\text {multi }}\right)$ and hence that $z=z_{j}$ for $j=1, \ldots, M$. We find numerically that this results in a more stable reconstruction algorithm than the typical approach.

To calculate this angle, introduce

$$
\hat{\mathcal{J}}_{\text {multi }}(z):=\frac{\left(\hat{\Phi}_{\omega}(y, z), P_{\hat{N}_{\text {multi }}} \hat{\Phi}_{\omega}(y, z)\right)_{L^{2}\left(\Gamma_{i}\right)}}{\left\|\hat{\Phi}_{\omega}(y, z)\right\|_{L^{2}\left(\Gamma_{i}\right)}\left\|P_{\hat{N}_{\text {multi }}} \hat{\Phi}_{\omega}(y, z)\right\|_{L^{2}\left(\Gamma_{i}\right)}},
$$

where $P_{\hat{N}_{\text {multi }}}$ is the projection operator onto the range of $\hat{N}_{\text {multi }}$. Note that $\left|\hat{\mathcal{J}}_{\text {multi }}\right| \leq 1$ with equality if and only if $\hat{\Phi}_{\omega}(z) \in \operatorname{Range}\left(\hat{N}_{\text {multi }}\right)$. Then the angle between the $\hat{\Phi}_{\omega}(z)$ and 
the range of the near field operator is $\hat{I}_{\text {multi }}(z)=\arccos \left(\operatorname{Re}\left(\hat{\mathcal{J}}_{\text {multi }}(z)\right)\right)$. As the numerical results demonstrate in Section $4,\left(\hat{I}_{\text {multi }}(z)\right)^{-1}$ is large if and only if $z=z_{j}$. Note that $\hat{\mathcal{J}}_{\text {multi }}$ is similar in form to the indicator function introduced for time harmonic scattering in [27]. However, the two functions are derived in a very different fashion - the indicator function in [27] is not related to the range of the near field operator - and as far as the authors can tell, their similarity is only coincidental.

The range test in the frequency domain formulated in Theorem 2 can now be used to obtain a range test for time domain scattering.

Theorem 3. Assume $D=\left\{z_{1}, z_{2}, \cdots z_{M}\right\}$ and that $\Gamma_{i}$ and $\Gamma_{m}$ (not necessarily the same) are closed curves or open subsets of analytic curves which do not intersect $D$. Define $\ell_{z, \tau}^{\xi}(x, t)$ as in (12) with $z \in \mathbb{R}^{d}$ and $\tau>0$ and $\xi \in C_{0}^{\infty}\left(\mathbb{R}^{+}\right)$. Then $\ell_{z, \tau}^{\xi} \in \operatorname{Range}\left(N_{\text {multi }}\right)$ if and only if $z \in\left\{z_{1}, \ldots, z_{M}\right\}$, where $N_{\text {multi }}$ is given by (13).

Proof. Assume $\ell_{z, \tau}^{\xi} \in \operatorname{Range}\left(N_{\text {multi }}\right)$. This is true if and only if there exists some $g_{z, \tau}$ so that $\left(N_{\text {multi }} g_{z, \tau}\right)(y, t)=\ell_{z, \tau}^{\xi}(y, t)$, which by Parseval's equality is true if and only if

$$
\begin{aligned}
0 & =\int_{-\infty}^{\infty} e^{-2 \sigma t}\left\|\left(N_{\text {multi }} g_{z, \tau}\right)(y, t)-\ell_{z, \tau}^{\xi}(y, t)\right\|_{L^{2}\left(\Gamma_{i}\right)}^{2} \mathrm{~d} t, \quad \sigma>0 \\
& =\frac{1}{2 \pi} \int_{-\infty+i \sigma}^{\infty+i \sigma}\left\|\left(\hat{N}_{\text {multi }} \hat{g}_{z, \tau}\right)(y, \omega)-\widehat{\ell}_{z, \tau}^{\xi}(y, \omega)\right\|_{L^{2}\left(\Gamma_{i}\right)}^{2} \mathrm{~d} \omega .
\end{aligned}
$$

This holds true if and only if

$$
\left\|\left(\hat{N}_{\text {multi }} \hat{g}_{z, \tau}\right)(y, \omega)-\hat{\ell}_{z, \tau}^{\xi}(y, \omega)\right\|_{L^{2}\left(\Gamma_{i}\right)}^{2}=0, \quad \omega \in \mathbb{C}_{\sigma}
$$

Note that by analyticity $\hat{\ddot{\chi}}(\omega)=0$ and $\hat{\xi}(\omega)=0$ only for a discrete set of $\omega \in \mathbb{C}_{\sigma_{0}}$ with $\sigma>\sigma_{0}>0$. Hence, recalling (12) and (15) we now have that $\ell_{z, \tau}^{\xi} \in \operatorname{Range}\left(N_{\text {multi }}\right)$ if and only if

$$
\sum_{j=1}^{M} \alpha_{j} \hat{\Phi}_{\omega}\left(y, z_{j}\right)=\beta \hat{\Phi}_{\omega}(y, z), \quad y \in \Gamma_{i}, z \in \mathbb{R}^{d}, \omega \in \mathbb{C}_{\sigma_{0}} .
$$

where $\alpha_{j}=-m_{j} \widehat{\ddot{\chi}}(\omega) \int_{\Gamma_{m}} \hat{\Phi}_{\omega}\left(x, z_{j}\right) \hat{g}_{z, \tau}(x, \omega) \mathrm{d} s(x)$ and $\beta=\hat{\xi}(\omega) e^{i \omega \tau}$ But this is exactly the range test from Theorem 2 , and so is true if and only if $z \in\left\{z_{1}, \ldots, z_{M}\right\}$.

As in the frequency domain case, this leads to an inversion scheme for time dependent multistatic data. Indeed, to calculate the angle between $\Phi(y-z, t-\tau)$ and $\operatorname{Range}\left(N_{\text {multi }}\right)$, introduce

$$
\mathcal{J}_{\text {multi }}(z, \tau):=\frac{\left(\Phi(y-z, t-\tau), P_{N_{\text {multi }}} \Phi(y-z, t-\tau)\right)_{L^{2}\left(\Gamma_{i} \times \mathbb{R}\right)}}{\|\Phi(y-z, t-\tau)\|_{L^{2}\left(\Gamma_{i} \times \mathbb{R}\right)}\left\|P_{N_{\text {multi }}} \Phi(y-z, t-\tau)\right\|_{L^{2}\left(\Gamma_{i} \times \mathbb{R}\right)}},
$$

where $P_{N_{\text {multi }}}$ is the projection operator onto the range of $N_{\text {multi }}$. Then the angle between the $\Phi(y-z, t-\tau)$ and the range of the near field operator is $I_{\text {multi }}(z, \tau)=\arccos \left(\mathcal{J}_{\text {multi }}(z, \tau)\right)$. Note that, unlike in the frequency domain case, we do not need to take a the real part of 
$\mathcal{J}_{\text {multi }}$ since the time domain values are inherently real-valued. As seen in Section 4, the indicator function $I_{\text {multi }}(z, \tau):=\arccos \left(\mathcal{J}_{\text {multi }}(z, \tau)\right) \approx 0$ if and only if $z=z_{j}$.

It is not completely clear how the sampling time $\tau$ affects reconstructions. However, numerical examples in Section 4 suggest that its choice is not very important for scattering from small and weak scatterers. On the other hand, numerical experiments with large obstacles, for which the above theory is not justified, show that a good choice of $\tau$ results in reconstruction of both the shape and location of an object. Poor choice of $\tau$ for large objects only allows the reconstruction of the location of the objects.

\subsection{Reconstruction of point scatterers from patches of time do- main multistatic data}

A key point in the reduction of data collection requirements is that the above theorems make very weak assumptions about the geometry of $\Gamma_{i}$ and $\Gamma_{m}$. As such, both $\Gamma_{i}$ and $\Gamma_{m}$ can be chosen as, e.g., sectors of a circle with a very small aperture. However, because of errors in data collection and limitations in measurement accuracy, this is not feasible in practice. Nonetheless, numerical simulations suggest that a patch of multistatic data, in which $\Gamma_{i}=\Gamma_{m}$ are, e.g., sectors of a circle with a very small aperture gives some indication of the hidden objects.

These observations lead to a simple technique for limiting data collection requirements in obstacle reconstruction: collect multistatic data on a small patch array of transmitters and receivers, then repeatedly move the array around the obstacles and repeat the experiment. Once this data is collected, reconstruct the obstacles from each experiment and post-process these reconstructions to give one reconstruction incorporating each experiment. The sim-

plest post-processing is to compute a weighted average of each reconstruction, though more complex processes may be applied.

As is shown in Section 4, this patch data and post-processing step results in acceptable reconstructions. Indeed, in an error-free case it is theoretically justified. However, in practice it requires a possibly time consuming reconstruction process for each set of patch data. Each of these reconstructions may further require regularization and choice of regularization parameters. Furthermore, a simple average of each reconstruction does not take into account that reconstructions should be similar, as they come from the same objects. More sophisticated post-processing algorithms could certainly alleviate this problem, but we do not explore them here. In the next section, we propose a method which requires only one reconstruction using even less data. Unlike the multistatic patch method, however, we are unable to fully justify its theory.

\subsection{Reconstruction of point scatterers from time domain quasi- backscattering data}

For simplicity we assume that the transmitters are distributed on the boundary $\Gamma_{i}:=S_{R}$ of a large ball $B_{R}$ centered at the origin containing the scatterer $\bar{D} \subset B_{R}$ and for each transmitting point $y \in \Gamma_{i}$ the scattered field is measured at $\Gamma_{m}^{(y)}:=S_{R} \cap B_{\delta}(y)$, where $B_{\delta}(y)$ is a small ball centered at $y$ of radius $\delta$. We will first consider briefly the full backscattering 
case when $\delta \rightarrow 0$. As numerical results demonstrate below, the quasi-backscattering setting for $\delta>0$ produces better reconstructions than the full backscattering case.

Consider the weak scattering near field backscattering operator for $(y, t) \in \Gamma_{i} \times \mathbb{R}^{+}$,

$$
\begin{aligned}
\left(N_{\text {backscattering }} g\right)(y, t) & =\int_{\mathbb{R}} u^{s}(y, t-\tau ; y) g(\tau) \mathrm{d} \tau \\
& =-\sum_{j=1}^{M} \frac{m_{j}}{\left(4 \pi\left|y-z_{j}\right|\right)^{2}} \int_{\mathbb{R}} \ddot{\chi}\left(t-\tau-2\left|y-z_{j}\right|\right) g(\tau) \mathrm{d} \tau .
\end{aligned}
$$

Taking the Fourier-Laplace transform yields

$$
\begin{aligned}
\left(\hat{N}_{\text {backscattering }} g\right)(y, \omega) & =-\omega^{2} \sum_{j=1}^{M} \frac{m_{j}}{\left(4 \pi\left|y-z_{j}\right|\right)^{2}} \hat{\chi}(\omega) \hat{g}(\omega) \exp \left(2 i \omega\left|y-z_{j}\right|\right) \\
& =-\omega^{2} \sum_{j=1}^{M} m_{j} \hat{\chi}(\omega) \hat{g}(\omega) \hat{\Phi}_{\omega}^{2}\left(y, z_{j}\right) \\
& =\sum_{j=1}^{M} \alpha_{j}(\omega) \hat{\Phi}_{\omega}^{2}\left(y, z_{j}\right),
\end{aligned}
$$

where $\alpha_{j}(\omega)=-\omega^{2} m_{j} \hat{\chi}(\omega) \hat{g}(\omega)$ and $\omega \in \mathbb{C}_{\sigma_{0}}$ for $\sigma_{0}>0$. Notice the similarities between $\widehat{N}_{\text {backscattering }}$ and $\widehat{N}_{\text {multi }}$ and define the sampling function

$$
\psi_{z, \tau}^{\xi}(y, t)=\left(\mathcal{F}^{-1}\left[\hat{\Phi}_{\omega}^{2}(y, z)\right] * \xi(\cdot-\tau)\right)
$$

where $\xi \in C_{0}^{\infty}$ and $\mathcal{F}^{-1}$ here denotes the inverse of the Fourier-Laplace transform defined in Section 2. Using the same arguments as in the proof of Theorem 3 for the multistatic case, the form of $\widehat{N}_{\text {backscattering }}$ suggests that

$$
I_{\text {backscattering }}(z, \tau):=\arccos \left(\frac{\left(\psi_{z, \tau}^{\xi}(y, t), P_{N_{\text {backscattering }}} \psi_{z, \tau}^{\xi}(y, t)\right)_{L^{2}\left(\Gamma_{i} \times \mathbb{R}\right)}}{\left\|\psi_{z, \tau}^{\xi}(y, t)\right\|_{L^{2}\left(\Gamma_{i} \times \mathbb{R}\right)}\left\|P_{N_{\text {backscattering }}} \psi_{z, \tau}^{\xi}(y, t)\right\|_{L^{2}\left(\Gamma_{i} \times \mathbb{R}\right)}}\right)
$$

acts as an indicator for the location of $z_{j}$, where $P_{N_{\text {backscattering }}}$ is the projection operator onto the range of $N_{\text {backscattering }}$.

As shown in Figure 7 , reconstructions using $I_{\text {backscattering }}$ are quite recognizable, but less clear than their quasi-backscattering equivalents. Hence, we suggest collecting quasibackscattering data with $\delta>0$. This differs from the results originally introduced for the quasi-backscattering context in $[24,26]$, in which a specific relationship between $\Gamma_{i}$ and $\Gamma_{m}^{(y)}$ is required in order to reconstruct $z_{j}$ under the assumption that $\left|x-z_{j}\right| \gg 1$. Here we do not require any such relationship or that data is collected sufficiently-far from the point scatterers. However, unlike in [24, 26], we are unable to prove an exact characterization of which components of $\left\{z_{j}\right\}$ can be reconstructed. Indeed, other than an intuitive argument that more data leads to better reconstructions, we are unsure why the quasi-backscattering reconstructions are superior to the backscattering reconstructions. 
The quasi-backscattering time domain weakly scattering near field operator $N_{\text {quasi }}$ : $H_{\sigma}^{p}\left(\mathbb{R}^{+}, L^{2}\left(S_{R}\right)\right) \rightarrow H_{\sigma}^{p}\left(\mathbb{R}^{+}, L^{2}\left(S_{R}\right)\right), \sigma>0, p \in \mathbb{R}$ is defined by

$$
\left(N_{\text {quasi }} g\right)(y, t)=\int_{\mathbb{R}} \int_{\Gamma_{m}^{(y)}} u_{B}^{s}(x, t-\tau ; y) g(x, \tau) \mathrm{d} s(x) \mathrm{d} \tau, \quad(y, t) \in \Gamma_{i} \times \mathbb{R}^{+} .
$$

For a collection of point sources with strength $m_{j}$ centered at points $z_{j}, j=1, \ldots, M, N_{\text {quasi }}$ takes the form

$$
\left.\left(N_{\text {quasi }} g\right)(y, t)=-\sum_{j=1}^{M} m_{j}\left(\Phi\left(z_{j}-y, \cdot\right) *\left(S_{\Gamma_{m}^{(y)}}^{\ddot{\chi}} g\right)\right)\left(z_{j}, \cdot\right)\right)(t), \quad(y, t) \in \Gamma_{i} \times \mathbb{R}^{+}
$$

and the Fourier-Laplace transform of the quasi-backscattering near field equation reads

$$
\sum_{j=1}^{M} m_{j} \widehat{\ddot{\chi}}(\omega) \hat{\Phi}_{\omega}\left(y, z_{j}\right) \int_{\Gamma_{m}^{(y)}} \hat{\Phi}_{\omega}\left(x, z_{j}\right) g(x) \mathrm{d} s(x)=-\hat{\xi}(\omega) e^{i \omega \tau} \hat{\Phi}_{\omega}^{2}(y, z) \quad y \in \Gamma_{i}, z \in \mathcal{Z}
$$

for $\omega \in \mathbb{C}_{\sigma_{0}}, \sigma>\sigma_{0}>0$. Unfortunately, as opposed to the backscattering or multistatic cases, in (21) the term

$$
J(y):=\int_{\Gamma_{m}^{(y)}} \hat{\Phi}_{\omega}\left(x, z_{j}\right) g(x) \mathrm{d} s(x)
$$

is not proportional to $\hat{\Phi}_{\omega}\left(y, z_{j}\right)$. As such, the arguments used to justify Theorem 2 no longer apply. We would like to show that $J(y)=c \hat{\Phi}_{\omega}\left(y, z_{j}\right)+O(\delta)$ where $c$ is independent of $y$. To this end, for a small patch $\Gamma_{m}^{(y)}$ on the sphere of radius $R$, say with diameter $\delta>0$, where $\delta$ is small compared to $\left|y-z_{j}\right|$ for $j=1, \ldots, M$, up to order $\delta^{2}$ we can replace $\Gamma_{m}^{(y)}$ by the tangent plane in $\mathbb{R}^{3}$ or line in $\mathbb{R}^{2}$ at $y$; let us denote it by $T_{y}^{\delta}$. Hence $x \in T_{y}^{\delta}$ and we have that $x=y+\eta y^{\perp}$ where $0<\eta<\delta$ and $y^{\perp}$ are the components of $y$ parallel to the tangent plane. Simple asymptotic calculations, which for sake of the argument, we present here only in the $\mathbb{R}^{3}$ case, give

$$
\begin{aligned}
& \left|x-z_{j}\right|=\left|y-z_{j}\right|+\eta \frac{\left(z_{j} \cdot y^{\perp}\right)}{\left|y-z_{j}\right|}+O\left(\delta^{2}\right) \text { and } \\
& \left|x-z_{j}\right|^{-1}=\left|y-z_{j}\right|^{-1}\left(1-\eta \frac{\left(z_{j} \cdot y^{\perp}\right)}{\left|y-z_{j}\right|^{2}}+O\left(\delta^{2}\right)\right)
\end{aligned}
$$

and hence, up to order $O\left(\delta^{2}\right)$

$$
J(y) \approx \hat{\Phi}_{\omega}\left(y, z_{j}\right) \int_{\left|\eta y^{\perp}\right|<\delta} e^{\frac{i \omega \eta\left(z_{j} \cdot y^{\perp}\right)}{\left|y-z_{j}\right|}}\left(1-\eta \frac{\left(z_{j} \cdot y^{\perp}\right)}{\left|y-z_{j}\right|^{2}}\right) g\left(y+\eta y^{\perp}\right) d\left(y+\eta y^{\perp}\right) .
$$

Hence we obtain the desired result if $\left(z_{j} \cdot y^{\perp}\right)=0$ for all $y \in \Gamma_{i}$ and $j=1, \ldots, M$. There is a special measurement configuration which works only in $\mathbb{R}^{3}$ and is detailed in [24]. Here, the measurement geometry is specified so that the integral in the above equation is independent of $y$. Indeed, in this set-up, $\Gamma_{i}$ is set to be a line parallel to some direction $\hat{v}$ and for each 
$y \in \Gamma_{i}$, the corresponding measurements are taken on a line parallel to $\hat{v}^{\perp}$ passing through $y$. In this set-up,

$$
\sum_{j=1}^{M} m_{j} \widehat{\ddot{\chi}}(\omega) \hat{\Phi}_{\omega}^{2}\left(y, z_{j}\right)=-\hat{\xi}(\omega) e^{i \omega \tau} \hat{\Phi}_{\omega}^{2}(y, z) \quad y \in \Gamma_{i}, z \in \mathcal{Z}
$$

provides an exact range test for $z_{j}$ whose projections to $\hat{v}^{\perp}$ differ. See Theorem 1 in [24] for more details. In the far field this setup becomes simpler and is studied in [26]. Nevertheless, for our data configuration in both $\mathbb{R}^{2}$ and $\mathbb{R}^{3},(21)$ shows that if $z=z_{j}$ for some $j=1, \ldots, M$, then we can find a $g:=g_{z_{j}, y} \in L^{2}\left(T_{y}^{\delta}\right)$ that solves exactly (21). Arguing heuristically, taking $g$ as an approximating sequence of the Dirac delta function at $y$ suggest that a range test as in the framework built up in the previous sections allowing us to introduce an indicator function for finding $z_{j}$.

As the numerical examples below demonstrate, these assumptions on the geometry of $\Gamma_{m}$ and $\Gamma_{i}$ do do not seem to be active. Indeed, based on the discussion above, after taking the inverse Fourier-Laplace transform of (21), we introduce an indicator function to measure the angle between $\psi_{z, \tau}$ and Range $\left(N_{\text {quasi }}\right)$. Let

$$
\mathcal{J}_{\text {quasi }}(z, \tau):=\frac{\left(\psi_{z, \tau}^{\xi}(y, t), P_{N_{\text {quasi }}} \psi_{z, \tau}^{\xi}(y, t)\right)_{L^{2}\left(\Gamma_{i} \times \mathbb{R}\right)}}{\left\|\psi_{z, \tau}^{\xi}(y, t)\right\|_{L^{2}\left(\Gamma_{i} \times \mathbb{R}\right)}\left\|P_{N_{\text {quasi }}} \psi_{z, \tau}^{\xi}(y, t)\right\|_{L^{2}\left(\Gamma_{i} \times \mathbb{R}\right)}},
$$

where $P_{N_{\text {quasi }}}$ is the projection operator onto the range of $N_{\text {quasi }}$. We demonstrate in Section 4 that the indicator function $I_{\text {quasi }}(z, \tau):=\arccos \left(\mathcal{J}_{\text {quasi }}(z, \tau)\right) \approx 0$ if and only if $z=z_{j}$.

In exactly the same way, we can derive a time harmonic indicator function for quasibackscattering data. In particular, time harmonic reconstructions are computed with

$$
\hat{I}_{\text {quasi }}(z)=\arccos \left(\operatorname{Re}\left(\frac{\left(\hat{\Phi}_{\omega}^{2}(y, z), P_{\hat{N}_{\text {quasi }}} \hat{\Phi}_{\omega}^{2}(y, z)\right)_{L^{2}\left(\Gamma_{i}\right)}}{\left\|\hat{\Phi}_{\omega}^{2}(y, z)\right\|_{L^{2}\left(\Gamma_{i}\right)}\left\|P_{\hat{N}_{\text {quasi }}} \hat{\Phi}_{\omega}^{2}(y, z)\right\|_{L^{2}\left(\Gamma_{i}\right)}}\right)\right) .
$$

We will show below that time dependent reconstructions out perform time harmonic reconstructions when an incident field is transmitted from only few points.

\subsection{Linear sampling method for extended objects under the time domain Born model}

Before showing numerical reconstructions of point objects, we discuss one technique, the linear sampling method, for extending the above multistatic results to extended obstacles which are weakly scattering. Full justification the linear sampling for the weak scattering case - as well as full justification under a strongly scattering model - requires new results about an associated interior transmission problem which we are unable to prove.

To introduce the linear sampling method for small objects, we follow [20], in which the linear sampling method for strongly scattering time domain data for inhomogeneous media is first investigated. The primary idea in linear sampling is to find a regularized solution to the near field equation (10). Then $I_{\mathrm{LSM}}(z, \tau)=\left\|g_{z, \tau}\right\|_{L^{2}\left(D, \mathbb{R}^{+}\right)}^{-1}$ is used as an indicator 
function. The values $I_{\mathrm{LSM}}$ are then used to suggest where $D$ is located; the $z \in \mathbb{R}^{d}$ so that $I_{\mathrm{LSM}}(z)$ is large are our reconstruction of $D$.

By making use of the volume integral equation representation of $u_{B}^{s}$, we can factor $N_{\text {multi }}$ into the product of two well-studied operators. In particular,

$$
\begin{aligned}
\left(N_{\text {multi }} g\right)(y, t) & =\int_{\mathbb{R}} \int_{\Gamma_{m}} u_{B}^{s}(x, t-\tau ; y) g(x, \tau) \mathrm{d} s(x) \mathrm{d} \tau \\
& =-\int_{\mathbb{R}} \int_{\Gamma_{m}}\left(\int_{\mathbb{R}} \int_{D} m(z) \Phi(x-z, t-\tau-s) u_{t t}^{i}(z, s, y) \mathrm{d} V(z) \mathrm{d} s\right) g(x, \tau) \mathrm{d} s(x) \mathrm{d} \tau \\
& =-\int_{\mathbb{R}} \int_{D} m(z) u_{t t}^{i}(z, s ; y) \int_{\mathbb{R}} \int_{\Gamma_{m}} \Phi(x-z, t-\tau-s) g(x, \tau) \mathrm{d} s(x) \mathrm{d} \tau \mathrm{d} V(z) \mathrm{d} s \\
& =-\int_{D} m(z)\left(\Phi(z-y, \cdot) * \ddot{\chi}(\cdot) *\left(S_{\Gamma_{m}} g\right)(z, \cdot)\right)(t) \mathrm{d} V(z) \\
& =-\int_{\mathbb{R}} \int_{D} m(z) \Phi(z-y, t-\tau)\left(S_{\Gamma_{m}}^{\ddot{\chi}} g\right)(z, \tau) \mathrm{d} V(z) \mathrm{d} \tau .
\end{aligned}
$$

$S_{\Gamma_{m}}^{\ddot{\chi}}$ is defined by (14).

This yields the following factorization

$$
N_{\text {multi }} g=\gamma_{\Gamma_{i}} G S_{\Gamma_{m}}^{\ddot{\chi}} g
$$

where $G$ is the wave equation solution operator defined by (5), and $\gamma_{\Gamma_{i}}$ is the trace operator restricting the solution to $\Gamma_{i}$. Using (6), and combining results for the multiple scattering linear sampling theory [20] with the Born transmission eigenvalue results in [13], it can be shown that the weakly scattering near field operator

$$
N_{\text {multi }}: H_{\sigma}^{p}\left(\mathbb{R}^{+}, L^{2}\left(\Gamma_{m}\right)\right) \rightarrow H_{\sigma}^{p}\left(\mathbb{R}^{+}, H^{1 / 2}\left(\Gamma_{i}\right)\right), \quad p \in \mathbb{R} \text { and } \sigma>0
$$

is bounded, injective, and has dense range. Note that when $p=0$ we have the mapping properties used above. By a contradiction argument it is also easy to show as in [20] that for $z \notin D$ any approximate solution of (10) is such that $\left\|g_{z, \tau}\right\|_{H_{\sigma}^{p}\left(\mathbb{R}^{+}, L^{2}\left(\Gamma_{m}\right)\right)}$ blows up as the regularization parameter in the equation goes to zero. However a complete justification of the linear sampling method, namely to describe the behavior of the approximate solution of (10) for $z \in D$, one need to find causal solution to the so-called interior transmission problem for the Born problem. This problem is to find $w(x, t)$ and $v(x, t)$ satisfying

$$
\begin{array}{cl}
w_{t t}(x, t)-\Delta w(x, t)=-m(x) v & (x, t) \in D \times \mathbb{R}^{+} \\
v_{t t}(x, t)-\Delta v(x, t)=0 & (x, t) \in D \times \mathbb{R}^{+} \\
w(x, t)=\ell_{z, \tau}^{\xi}(x, t) & (x, t) \in \partial D \times \mathbb{R}^{+} \\
\frac{\partial w(x, t)}{\partial \nu}=\frac{\partial \ell_{z, \tau}^{\xi}(x, t)}{\partial \nu} & (x, t) \in \partial D \times \mathbb{R}^{+} \\
w(x, 0)=w_{t}(x, 0)=v(x, 0)=v_{t}(x, 0)=0 & x \in D .
\end{array}
$$

The solution of this problem remains open both for the Born approximation model and the full multiple scattering model. Fourier or Fourier-Laplace analysis fail to work for this problem since the transformed homogeneous problem (known as the transmission eigenvalue 
problem) is non-selfadjoint and its complex eigenvalues may have unbounded imaginary part. Moreover, there are either infinitely many real eigenvalues or a sequence of complex eigenvalues may approach the real axis $[13,33]$. Despite a significant amount of research on the transmission eigenvalue problem (see [12] and references therein), optimal bounds on transmission eigenvalues have not yet been established. However, numerical implementation of the linear sampling method, i.e. finding a regularized solution of (10) shows that $\left(\left\|g_{z, \tau}\right\|_{H_{\sigma}^{p}\left(\mathbb{R}^{+}, L^{2}\left(\Gamma_{m}\right)\right)}\right)^{-1}$ remains finite inside $D$ and is very small outside $D$. As such, the linear sampling method can be applied to image weak scatterers, as demonstrated in Figure 2 .

\section{Numerical Reconstructions}

To simulate forward scattering, we use the convolution quadrature-volume integral equation approach introduced in [30]. We use a Galerkin semi-discretization in space, rather than the collocation method suggested in [30]. With this, we simulate values of $u^{s}\left(x_{j}, t_{k} ; y_{i}\right)$ at some discrete values $x_{j} \in \Gamma_{m}, y_{i} \in \Gamma_{i}$, and at $t_{k} \in[0, T]$, where $T>0$ is some final time. We choose $T$ so that at least $99 \%$ of the energy of $u^{s}$ has left the computational domain. For simplicity, we always take $n=1$ outside of $D$ and $n=1.1$ inside of $D$.

Using these simulations we can calculate discrete approximations to $N_{\text {multi }}$ and $N_{\text {quasi }}$, which we denote as $\mathbb{N}_{\text {multi }}$ and $\mathbb{N}_{\text {quasi }}$, respectively. As we explain below, we are more interested in a partial singular value decomposition (SVD) of these near field operators than in the matrices themselves. Since the near field operator is a convolution, this decomposition can be calculated quickly and without explicitly forming $\mathbb{N}_{\text {multi }}$ or $\mathbb{N}_{\text {quasi }}$ by using a fast Fourier transform, as described in [25].

In order to avoid inverse crimes, we calculate the full multiple scattering data, not the Born approximation to this data. We further avoid inverse crimes by adding noise to reconstructions, replacing $u^{s}$ with $(1+\epsilon \rho) u^{s}$ where $\epsilon$ is what we refer to below as the noise level and $\rho$ is a uniform random variable in $[-1,1]$. Our reconstructions use 10-25 transmitters. This less than one-half of the amount of transmitter locations as are typically used in sampling-type schemes, though our reconstructions are of similar accuracy. This is likely both due to the increased amount of a priori data we assume about our obstacles and the fact that time domain data contains more information than the single frequency time harmonic data which is usually used.

Unless otherwise noted in figure captions, obstacles are indicated by black lines. Red dots indicate location of transmitters and black dots the location of the receivers for each transmitter. Time domain data was simulated for 18 seconds with 600 time steps and with the impulse function $\chi(t)=\sin (4 t) \exp \left(-1.6(t-3)^{2}\right)$. All figures have $5 \%$ added noise. All reconstructions are rescaled to $[0,1]$ for comparison purposes.

\subsection{MUSIC and LSM reconstruction with multistatic data}

We first use $I_{\text {multi }}$ and $I_{\mathrm{LSM}}$ to find reconstructions using multistatic data. To this end, we compute $\mathbb{N}_{\text {multi }}=\mathbb{U S V}^{*}$, the SVD of $\mathbb{N}_{\text {multi }}$. Then, it is well-known that the projection operator can be written as $P_{\mathbb{N}_{\text {multi }}}=\mathbb{N}_{\text {multi }}\left(\mathbb{N}_{\text {multi }}^{*} \mathbb{N}_{\text {multi }}\right)^{-1} \mathbb{N}_{\text {multi }}^{*}=\mathbb{U S}\left(\mathbb{S}^{*} \mathbb{S}\right)^{-1} \mathbb{S}^{*} \mathbb{U}$, so long as 
each of the inverse matrices exists. By using the singular value decomposition, we avoid the need to construct the possibly-large matrix $\mathbb{N}_{\text {multi }}$ and can easily regularize using a spectral cut-off method, by looking for a gap in the singular values on the diagonal of $\mathbb{S}$ and using only the large singular values in reconstruction. We calculate $I_{\text {multi }}(z, \tau)$ in this way in order to reconstruct $D$. We calculate $I_{\text {LSM }}$ by solving (10) with a truncated SVD as in [20].

We calculate these multistatic indicator functions for two geometries in $\mathbb{R}^{2}$. This is shown in Figure 2, where forward data is calculated for 10 incident points and 10 measurement points. For the point obstacles on the right, we use a sampling time of $\tau=1$, though the reconstructions seem acceptable for any $0<\tau<T$. For the larger obstacle on the left, we set $\tau=5.4$. By choosing $\tau$ in this way, we reconstruct both the location and shape of $D$, while some values of $\tau$ only reconstruct the location. It is also an open problem to automatically chose $\tau$ for linear sampling methods under the full multiple scattering model. In these figures, large values indicate the reconstructed location of objects.
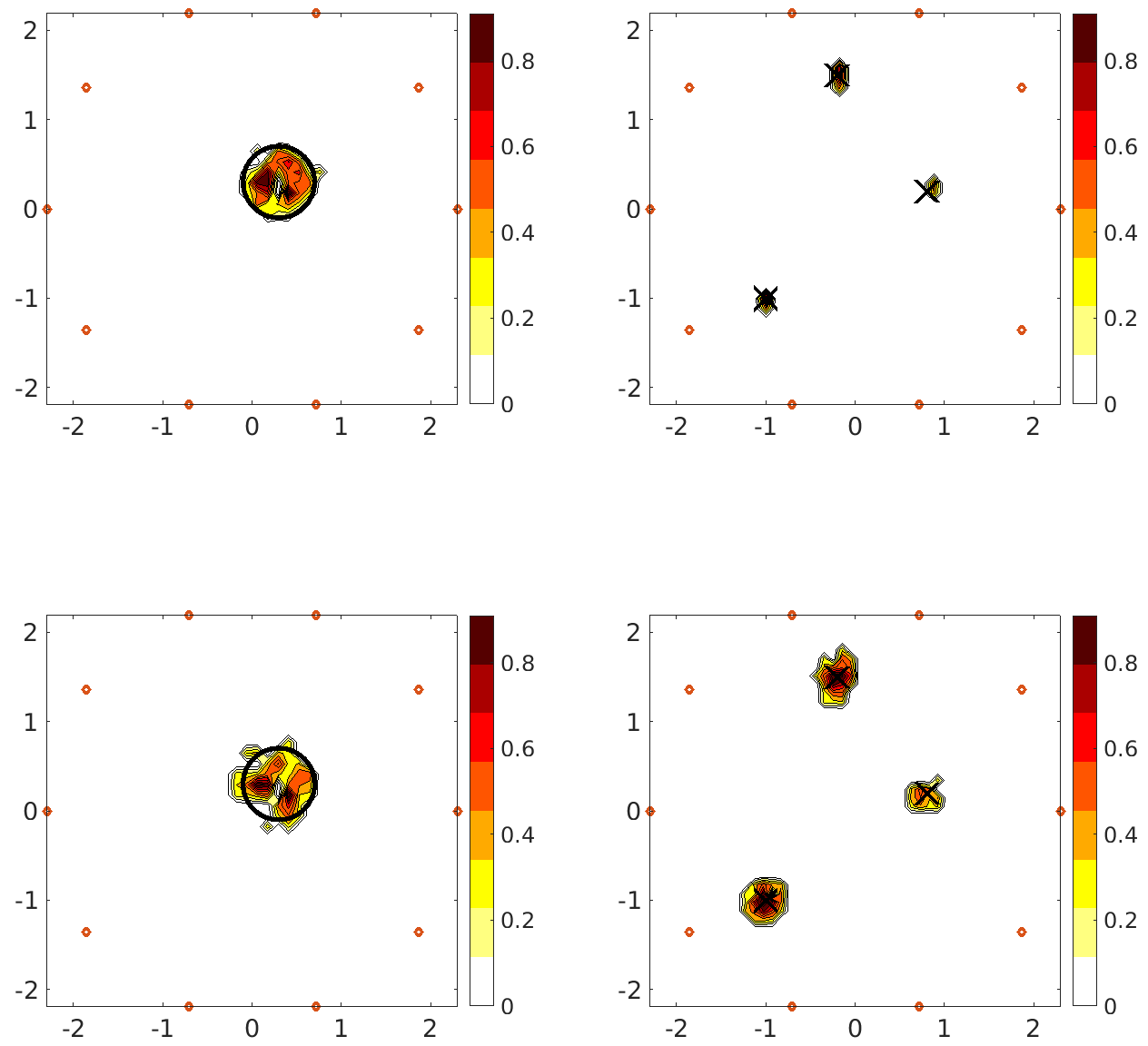

Figure 2: Plots of $\left(I_{\mathrm{LSM}}(z)\right)^{-1}$ (top) and $\left(I_{\text {multi }}(z)\right)^{-1}$ (bottom) for two different geometries.

We now restrict our figures to the averages of small multistatic patches. We demonstrate in Figure 3 that small objects and be reconstructed well if either the aperture of each patch 
is sufficiently large or if there are a large number of patches used. In Figure 4, we show the same experimental set-up used for larger objects, showing that reconstructions are worse, but still acceptable.
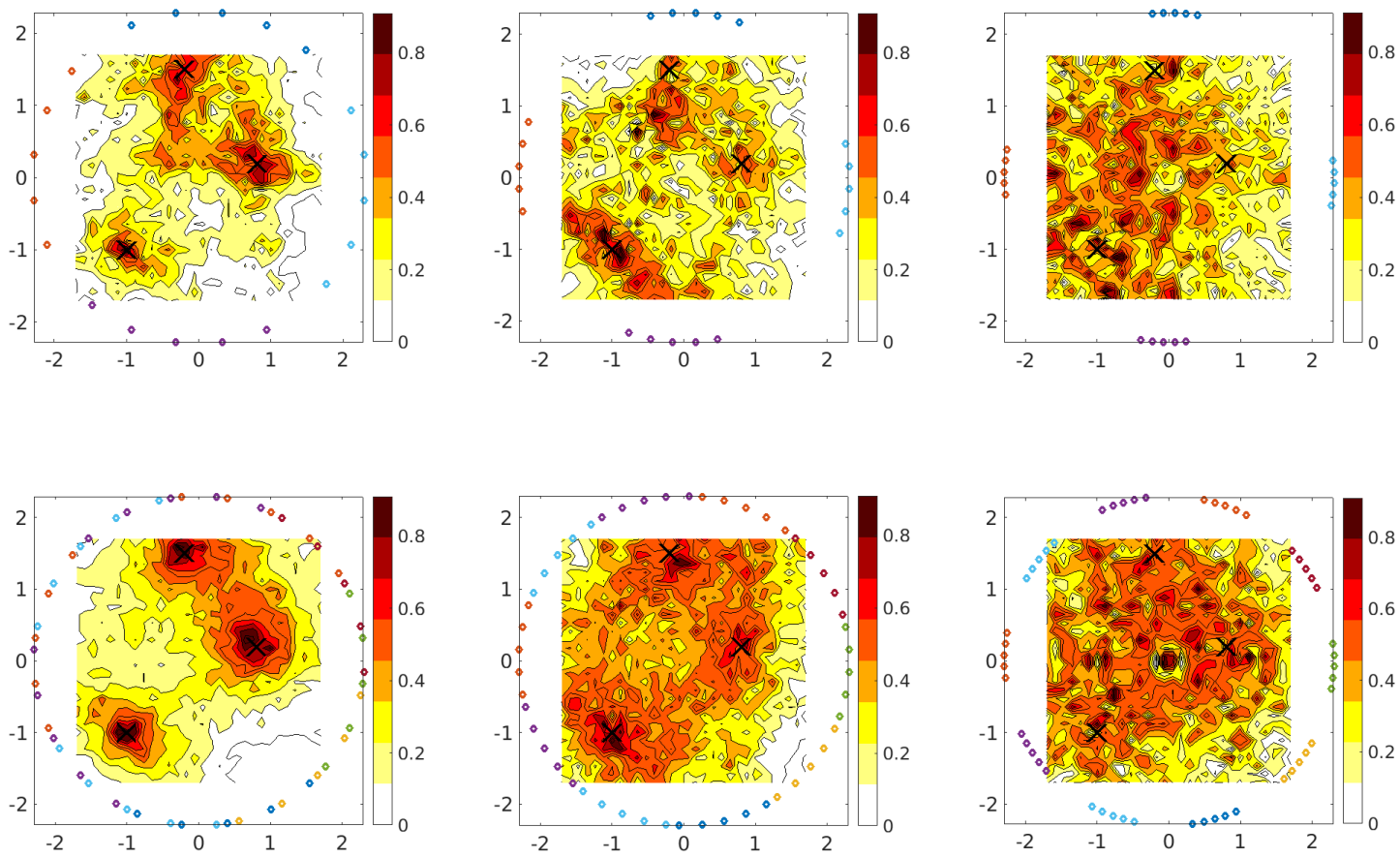

Figure 3: Multistatic patch reconstructions of the same geometry of small circles, indicated by black lines. (Top) Four patches are used with 5 transmitters and receivers each. From left-to-right, the aperture of each patch decreases from $\pi / 2$ to $\pi / 4$ to $\pi / 8$. (Bottom) The same experiment as top but with 10 patches. Each set of circles indicates the location of transmitters and receivers in each patch. Transmitters and receivers in the same patch each have the same color.

\subsection{Quasi-backscattering reconstructions}

In Figures 5-8 we use quasi-backscattering data to reconstruct the location of a number of objects. To construct $I_{\text {quasi }}$, we again use the SVD of the discrete near field operator to calculate the angle between the test functions and their projection onto the range of $\mathbb{N}_{\text {quasi }}$. We again regularize with a spectral cut-off.

In Figure 5 we demonstrate the feasibility of the proposed technique with different geometries. In this figure, we plot only the values of $\left(I_{\text {quasi }}(z)\right)^{-1}$ larger than a cut-off threshold chosen visually. In some ways, this figure then represents an idealized set of reconstructions. However, there are a number of algorithms which make this choice automatically, described for example in [10, 26]. For each figure, we use 20 transmitters, each with 4 receivers, and set $\delta=\pi / 100$. For each image, we use the temporal sampling point $\tau=1$, though as before this choice does not seem to seriously affect reconstructions. 

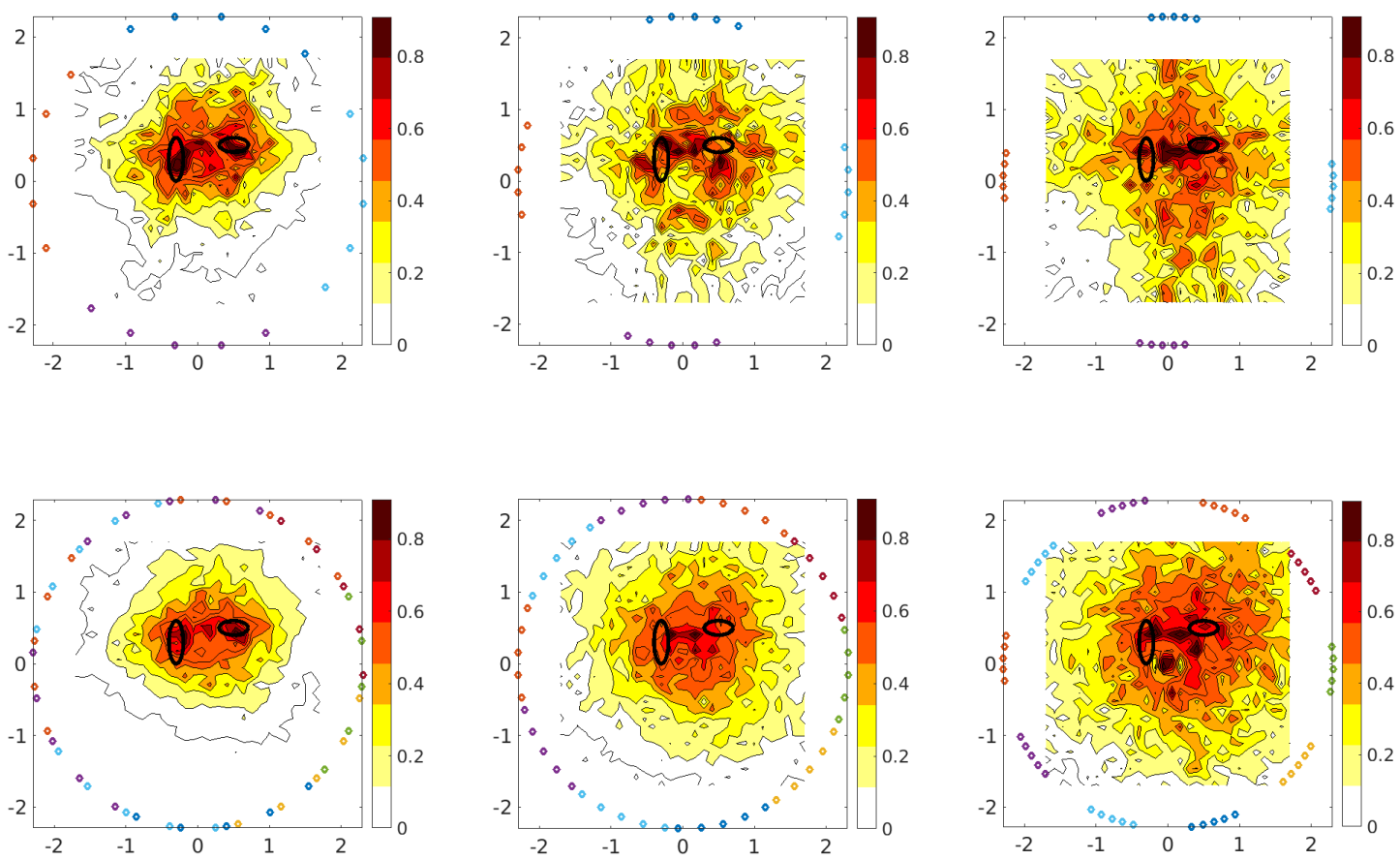

Figure 4: Multistatic patch reconstructions of the same geometry of medium-sized ellipses, indicated by black lines. (Top) Four patches are used with 5 transmitters and receivers each. From left-to-right, the aperture of each patch decreases from $\pi / 2$ to $\pi / 4$ to $\pi / 8$. (Bottom) The same experiment as top but with 10 patches. Each set of circles indicates the location of transmitters and receivers in each patch. Transmitters and receivers in the same patch each have the same color.

In Figure 6 we demonstrate the dependence of reconstructions on the number of transmitter locations. We show both time harmonic and time dependent reconstructions. As expected, reconstructions become more accurate when more transmitter locations are used. Furthermore, time dependent reconstructions are more accurate than time harmonic reconstructions, until a sufficient number of transmitters are used.

In the limiting case as $\delta \rightarrow 0$, the quasi-backscattering set-up becomes a pure backscattering set-up. In Figure 7 we show numerical examples of pure backscattering. There are a total of 30 transmitter locations, each with 1 receiver located at the same point. While reconstructions are not as sharp as, e.g., the full aperture multistatic reconstructions, there is a clear indication of object location.

Finally, we compare limited aperture reconstructions from multistatic data to limited aperture reconstructions from patch and quasi-backscattering data. As expected, Figure 8 demonstrates that the multistatic reconstructions are superior to the patch and quasibackscattering reconstructions, which are somewhat similar. Indeed, the quasi-backscattering algorithm results in reconstructions which are noisier and, the case of three point obstacles, only clearly reconstruct the two obstacles nearest the transmitter and receiver arrays, incorrectly indicating an extra obstacle in the bottom right. Patch data reconstructions are 

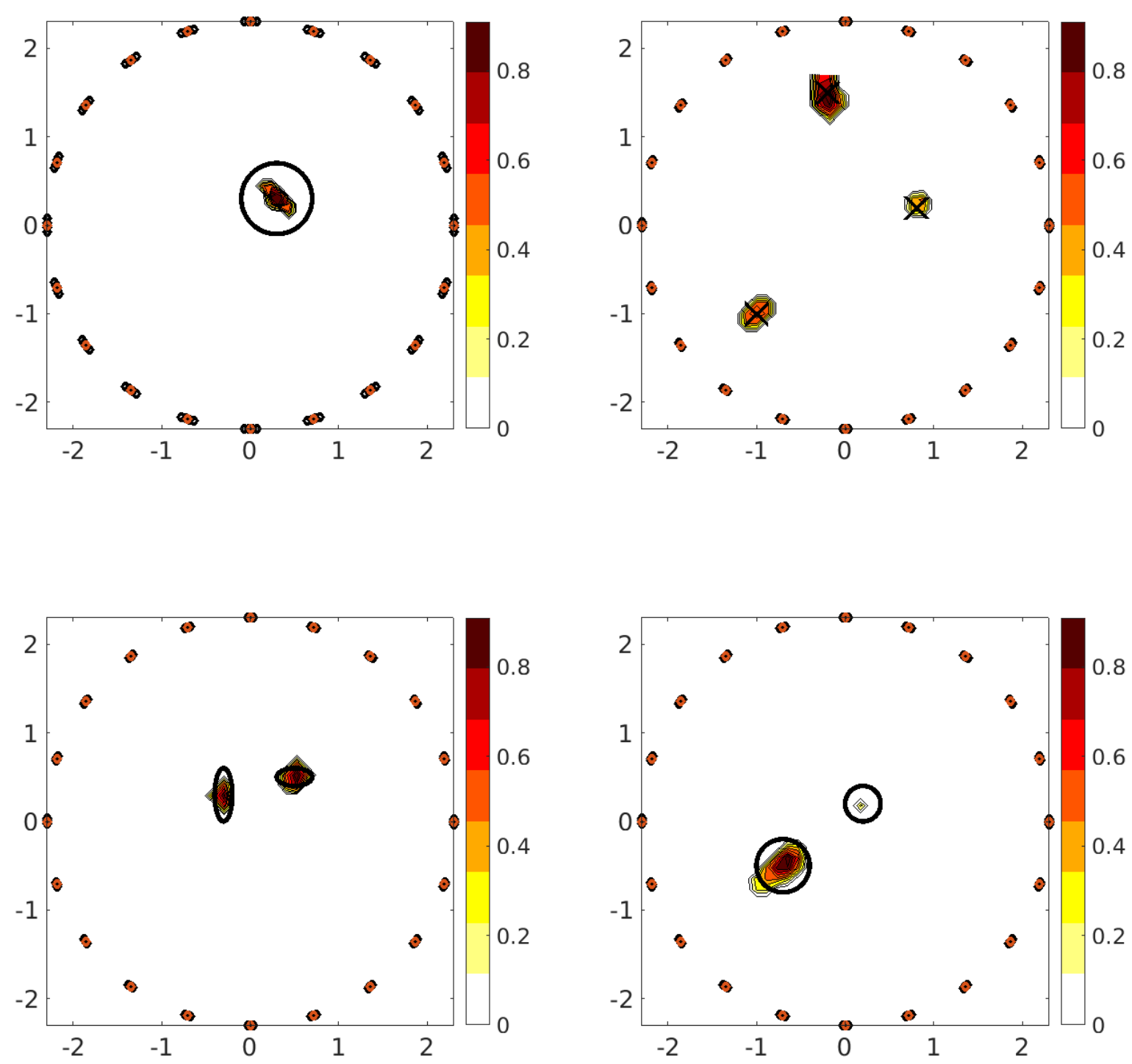

Figure 5: Plots of $\left(I_{\text {quasi }}(z, \tau)\right)^{-1}$ for four different geometries.

also noisier than multistatic reconstructions, but do not have the same issues as quasibackscattering reconstructions do with three point obstacles. The patch reconstructions do not separate the larger ellipses as effectively as the quasi-backscattering reconstructions. Note that both the multistatic and patch reconstructions required a careful selection of $\tau=5$ in order to produce optimal results, while the quasi-backscattering data did not need any such choice. 

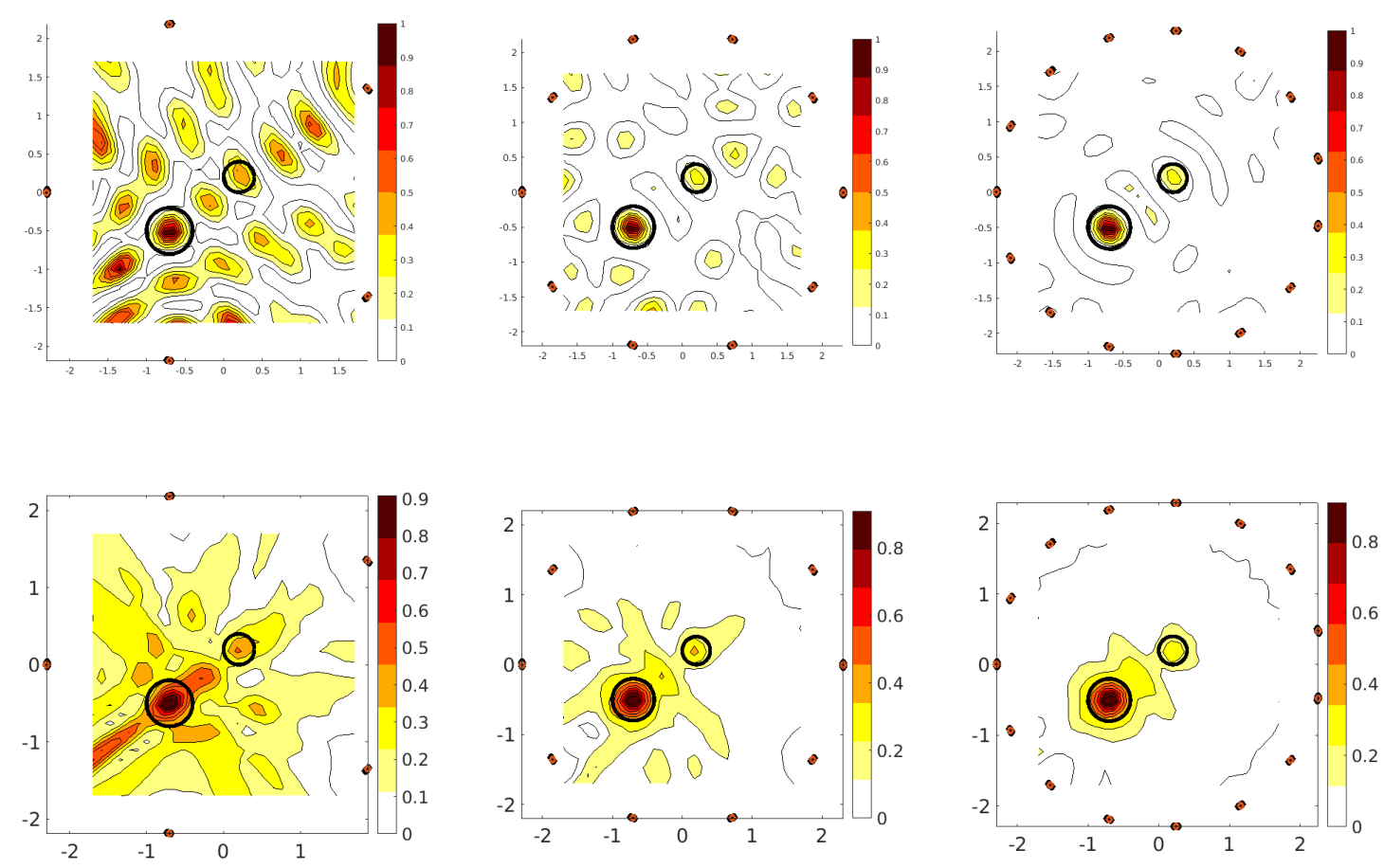

Figure 6: Plots of $\left(\hat{I}_{\text {quasi }}(z, \tau)\right)^{-1}$ (top) and $\left(I_{\text {quasi }}(z, \tau)\right)^{-1}$ (bottom), with a different number of transmitters in each row. On the left there are 5 transmitters, in the middle there are 10 transmitters, and the right there are 15 transmitters. Time harmonic data was computed with wavenumber $k=3$.

\section{Acknowledgments}

The research of F. Cakoni is supported in parts by AFOSR grant FA9550-17-1-0147, NSF Grant DMS-1602802 and Simons Foundation Award 392261. The research of J. Rezac is supported in part by NSF Grant DMS-1602802 and the University of Delaware Graduate Dissertation Fellowship. Both authors acknowledge the hospitality of the DeFI Team during their visit at École Polytechnique when part of this work was completed. Peter Monk is also thanked for his help with forward problem simulations.

\section{References}

[1] C.Y. Ahn, K. Jeon, and W.-K. Park, Analysis of MUSIC-type imaging functional for single, thin electromagnetic inhomogeneity in limited-view inverse scattering problem. Journal of Computational Physics 291 (2015) 198-217.

[2] H. Alqadah and N. Valdivia, A frequency based constraint for a multi-frequency linear sampling method. Inverse Problems 29 (9) (2013) 095019-095046. 

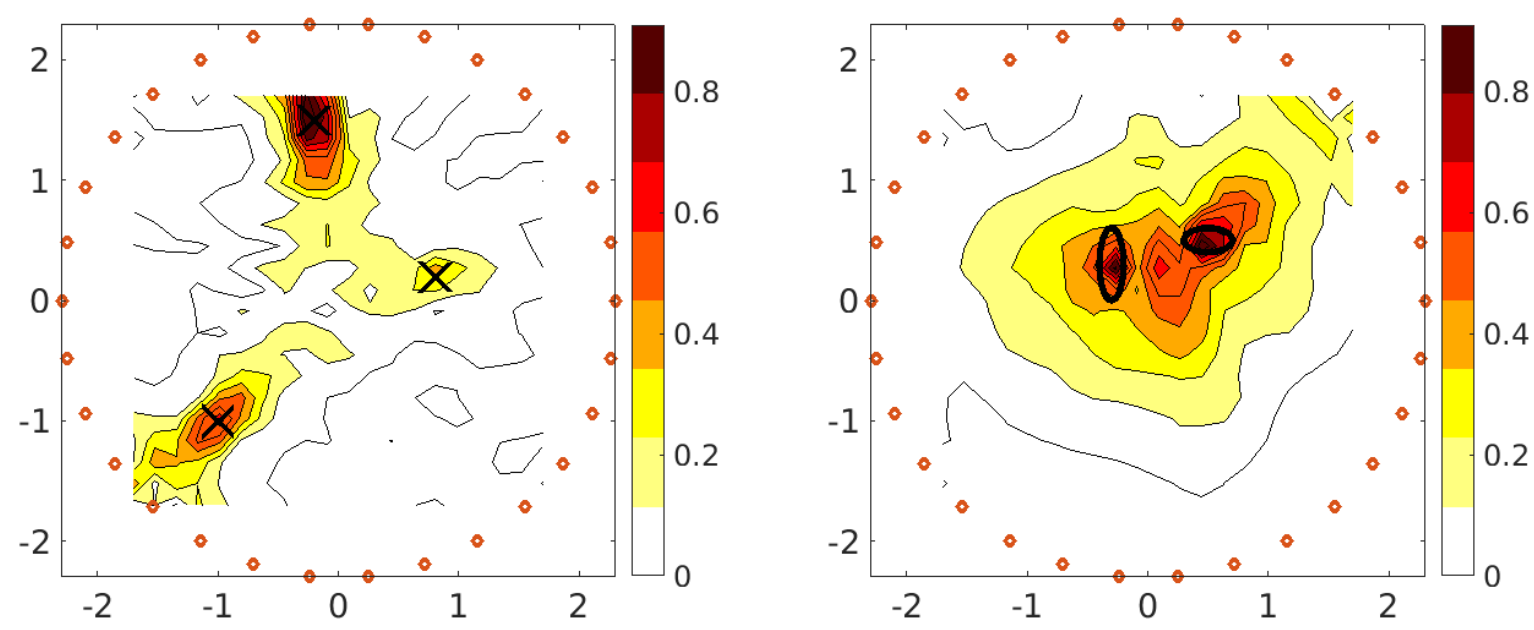

Figure 7: Backscattering reconstructions using $I_{\text {backscattering }}^{-1}(z, \tau)$ for two different geometries. In both figures, 30 transmitters are used and data is measured only at the location of the transmitter. Time domain data was simulated for 14 seconds with 480 time steps.

[3] H. Ammari, M. Asch, L. Guadarrama Bustos, V. Jugnon, and H. Kang, Transient wave imaging with limited-view data. SIAM Journal on Imaging Sciences, 4 (2011) 1097-1121.

[4] H. Ammari, E. Iakovleva and D. Lesselier, Two numerical methods for recovering small inclusions from the scattering amplitude at a fixed frequency. SIAM Journal of Scientific Computing, 27 (2007) 130-158.

[5] H. Ammari., J. Garnier, W. Jing, H. Kang, M. Lim, K. Sølna, and H. Wang, Mathematical and statistical methods for multistatic imaging, volume 2098 of Lecture Notes in Mathematics. Springer-Verlag (2013).

[6] H. Ammari, J. Garnier, H. Kang, M. Lim, K. Sølna, Multistatic imaging of extended targets. SIAM Journal on Imaging Sciences, 5 (2012) 564-600.

[7] H. Ammari, P. Garapon, L. Guadarrama Bustos, and H. Kang, Transient anomaly imaging by the acoustic radiation force. Journal of Differential Equations, 249 (2010) 1579-1595.

[8] H. Ammari and H. Kang, Reconstruction of small inhomogeneities from boundary measurements, volume 1846 of Lecture Notes in Mathematics. Springer-Verlag (2004).

[9] H. Ammari, H. Kang, E. Kim, M. Lim, and K. Louati, A Direct Algorithm for Ultrasound Imaging of Internal Corrosion. SIAM Journal of Numerical Analysis, 49 (3) (2011) 1177-1193. 
[10] R. Aramini, M. Brignone, J. Coyle, and M. Piana, Postprocessing of the Linear Sampling Method by Means of Deformable Models. SIAM Journal on Scientific Computing, 30 (5) (2008) 2613-2634.

[11] A. Bamberger and T. Ha Duong, Formulation variationnelle espace-temps pour le calcul par potentiel retardé de la diffraction d'une onde acoustique (I). Mathematical Methods in Applied Sciences, 8 (1986) 405-435.

[12] F. Cakoni, D. Colton and H. Haddar Inverse Scattering Theory and Transmission Eigenvalues. CBMS Series, SIAM Publications, 88 (2016).

[13] F. Cakoni, D. Colton and J.D. Rezac, The Born transmission eigenvalue problem. Inverse Problems, 32 (10) (2016) 105014-105028.

[14] Q. Chen, H. Haddar, A. Lechleiter and P. Monk, A sampling method for inverse scattering in the time domain. Inverse Problems, 26 (8) (2010) 085001-085018.

[15] A.J. Devaney, Super-resolution processing of multi-static data using time reversal and MUSIC. Unpublished paper, preprint available on the author's website (2000).

[16] M. Fink and C. Prada, Acoustic time-reversal mirrors. Inverse Problems, 17 (1) (2001) R1-R38.

[17] J.F. Funes, J.M. Perales, ML. Rapún, J.M. Vega, Defect detection from multifrequency limited data via topological sensitivity. Journal of Mathematical Imaging and Vision, 55 (19) (2016) 19-35.

[18] R. Griesmaier and C. Schmiedecke, A multifrequency MUSIC algorithm for locating small inhomogeneities in inverse scattering. Inverse Problems, 33 (3) (2017) 03501503132.

[19] Y. Guo, D. Hömberg, G. Hu, J. Li, H. Liu, A time domain sampling method for inverse acoustic scattering problems. Journal of Computational Physics, 314 (2016) 647-660.

[20] Y. Guo, P. Monk and D. Colton, Toward a time domain approach to the linear sampling method. Inverse Problems, 29 (9) (2013) 095016-095033.

[21] Y. Guo, P. Monk and D. Colton, The linear sampling method for sparse small aperture data. Applicable Analysis, 95 (8) (2015) 1599-1615.

[22] B. Guzina, F. Cakoni and C. Bellis, On the multi-frequency obstacle reconstruction via the linear sampling method. Inverse Problems, 26 (12) (2010) 125005-125035.

[23] T. Ha Duong, On retarded potential boundary integral equations and their discretization. From Topics in Computational Wave Propagation. Direct and Inverse Problems, ed P. Davies, P. Duncan, P. Martin, and B. Rynne. Springer (2003) 301-336. 
[24] H. Haddar and M. Lakhal, Identification of small objects with near-field data in quasibackscattering configuration. Advances in Acoustics and Vibration, Applied Condition Monitoring, 5 (2017) 43-54.

[25] H. Haddar, A. Lechleiter and S. Marmorat, An improved time domain linear sampling method for Robin and Neumann obstacles. Applicable Analysis, 93 (2) (2014) 369-390.

[26] H. Haddar and J.D. Rezac, A quasi-backscattering problem for inverse acoustic scattering in the Born regime. Inverse Problems, 31 (7) (2015) 075008-075016.

[27] K. Ito, B. Jin, and J. Zou, A direct sampling method to an inverse medium scattering problem. Inverse Problems, 28 (2) (2012) 025003-025014.

[28] A. Kirsch and N. Grinberg, The Factorization Method for Inverse Problems. Oxford University Press, 2008.

[29] A. Kirsch and A. Rieder, On the linearization of operators related to the full waveform inversion in seismology. Mathematical Methods in Applied Science, 37 (2014) 29953007.

[30] A. Lechleiter and P. Monk, The time-domain Lippmann-Schwinger equation and convolution quadrature. Numerical Methods for Partial Differential Equations, 31 (2) (2014) 517-540.

[31] W.-K. Park, Multi-frequency subspace migration for imaging of perfectly conducting, arc-like cracks in full- and limited-view inverse scattering problems. Journal of Computational Physics, 283 (2015) 52-80.

[32] Rakesh and G. Uhlmann, Uniqueness for the inverse backscattering problem for angularly controlled potentials. Inverse Problems, 30 (6) (2014) 065005-065029.

[33] J.D. Rezac, Direct methods for inverse scattering with time dependent and reduced data. Ph.D Thesis, University of Delaware, 2017.

[34] F.J. Sayas, Retarded Potentials and Time Domain Boundary Integral Equations: A Road Map. Springer Verlag, 2016.

[35] G. Uhlmann, A time-dependent approach to the inverse backscattering problem. Inverse Problems, 17 (4) (2001) 703716. 

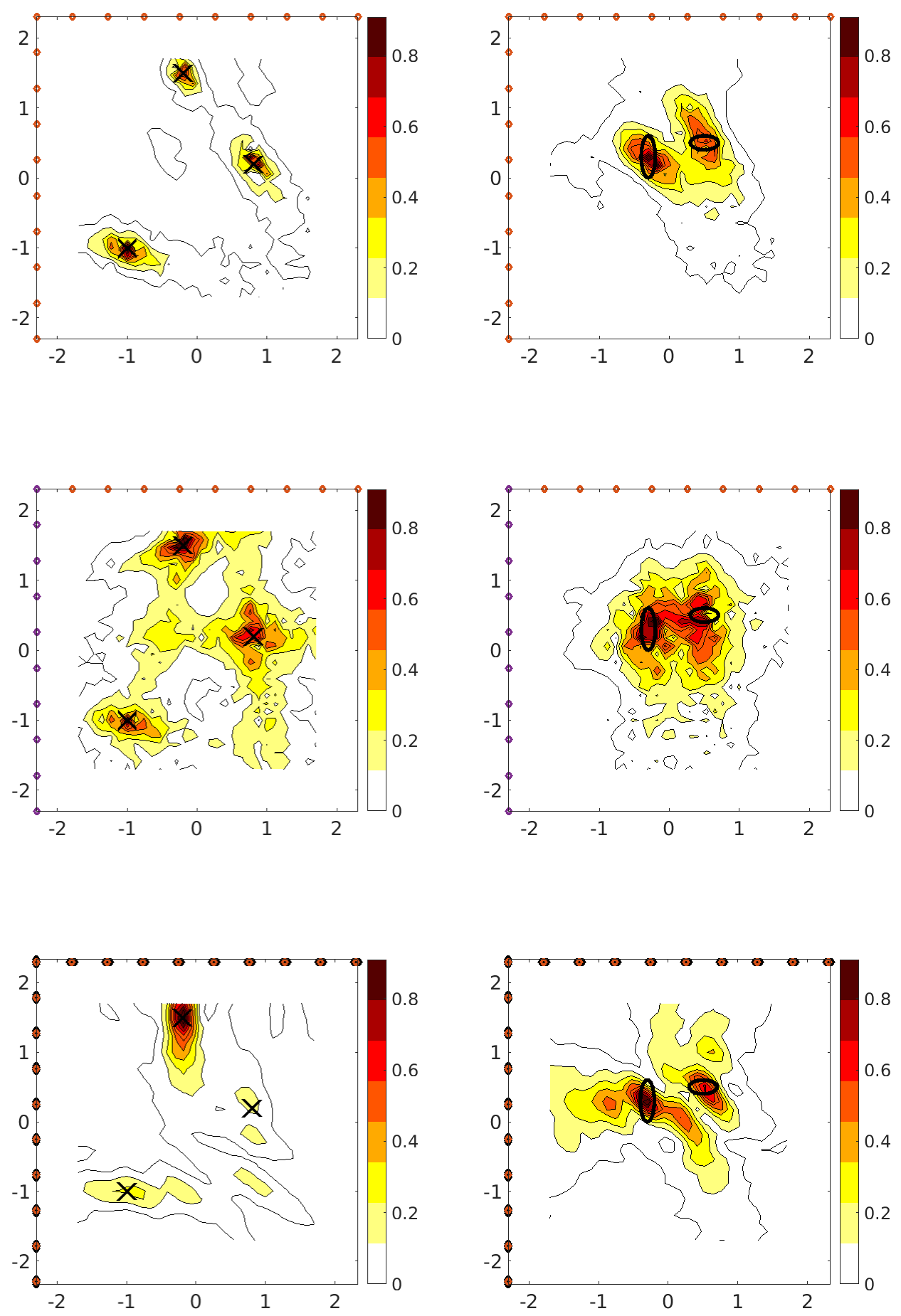

Figure 8: Limited aperture reconstructions using multistatic data with $I_{\text {multi }}^{-1}(z, \tau)$ (top), multistatic patch data with two patches (middle), and quasi-backscattering data with $I_{\text {quasi }}^{-1}(z, \tau)$ (bottom). In both figures, 19 transmitters are used and in the case of quasi-backscattering data, 4 receivers were used. 